\title{
Die postkeynesianische Ökonomik in der Finanzethik des katholischen Sozialethikers Bernhard Emunds - Diskussion aus einer angebotsorientierten Perspektive
}

Research Article

Felix Heider*

Received October 20, 2020; Accepted January 18, 2021

Zusammenfassung: Bernhard Emunds beschäftigt sich als deutschsprachiger katholischer Sozialethiker mit dem Thema der Finanzmärkte. Der vorliegende Beitrag stellt den Postkeynesianismus als sein ökonomisches Fundament vor. Danach werden die Schlussfolgerungen beschrieben, die Emunds für eine Regulierung von Finanzmärkten ableitet. Anschließend wird der Postkeynesianismus einer angebotsorientierten Sicht der Ökonomik gegenübergestellt. Zum Schluss wird die Frage behandelt, inwieweit der Postkeynesianismus ein geeignetes ökonomisches Fundament für die katholische Soziallehre sein kann.

Schlagwörter: Katholische Soziallehre • Regulierung • Finanzmärkte • Postkeynesianismus

JEL-KIassifikation: B59, G18

Abstract: Bernhard Emunds, a German Catholic social ethicist, is engaged in research into financial markets. This article introduces postKeynesianism as his economic foundation, followed by a description of conclusions Emunds draws about the regulation of financial markets. Thereafter, implications of the post-Keynesian framework are contrasted with those of supply-side economics. The article finally appraises the suitability of post-Keynesianism as an economic foundation for Catholic social teaching.

Keywords: Catholic Social Teaching • Regulation • Financial Markets • Postkeynesianism

\section{Finanzethik - ein Grenzgebiet zwischen Ethik und Wirtschaftswissenschaft}

Die Orientierung in moralischen Fragen, welche die katholische Soziallehre den Menschen vermitteln will, muss sich auch auf das Gebiet der Finanzmärkte erstrecken. Dieser Aufgabe widmet sich Bernhard Emunds, der am Oswald von Nell-Breuning-Institut für Wirtschafts- und Gesellschaftsethik in Frankfurt a.M. wirkt, in mehreren Veröffentlichungen. Im deutschen Sprachraum gibt es darüber hinaus kaum Vertreter der katholischen Soziallehre, die sich mit Finanzmärkten beschäftigen. Die Bedeutung der internationalen Finanzmärkte für das Leben der Menschen ist hoch: So gibt es Stimmen, die sagen, dass die gesamte ökonomische Entwicklung von der Finanzwirtschaft beherrscht wird (Stockhammer \& Ramskogler 2009: 332). Durch das Wechselspiel von Angebot und Nachfrage auf dem Finanzmarkt bildet sich der Preis des Geldes: der Zins. Jeder Wert eines Gutes, also jeder Preis, wird in Geldeinheiten ausgedrückt und damit vom Zins mitbestimmt. Der Einfluss, den Finanzmärkte auf die realwirtschaftliche Entwicklung haben, kann kaum überschätzt werden. Dies wird gerade in Zeiten einer Finanzkrise besonders deutlich spürbar (Sechrest 1993: 59-60). Folglich muss die Ethik 
diesen wichtigen Bereich des menschlichen Daseins in den Blick nehmen. Dementsprechend soll hier das Werk von Emunds einer tieferen Analyse unterzogen werden.

Eine Ethik der Finanzmärkte bzw. eine „Finanzethik“ ist nur vor dem Hintergrund einer wirtschaftswissenschaftlichen Positionsbestimmung möglich. Deswegen ist die Ethik der Finanzmärkte eine Schnittstelle von philosophischer Ethik und der Wirtschaftswissenschaft. Der Wissenschaftler muss in zwei Sphären gleichzeitig eine Position beziehen: Zum einen muss die Ethik ein Fundament haben und dies ist bei Emunds die katholische Soziallehre (Emunds 2014: 3-4). Zum anderen muss eine wirtschaftswissenschaftliche Positionierung vorgenommen werden. Diese von Emunds gewählte Position steht hier im Vordergrund: der sogenannte Postkeynesianismus.

In der zeitgenössischen Ökonomik nimmt der Postkeynesianismus eine Nebenrolle ein und betont selbst seine grundsätzlich eigenständige Position gegenüber dem ökonomischen „Mainstream“ (Walterskirchen 2016: 405-406). Emunds nimmt des Öfteren auf diese vom Mainstream abweichende Positionierung Bezug (z. B. Emunds 2014: 257, 273). Der Postkeynesianismus ist eine Wirtschaftstheorie, welche die Nachfrage besonders in den Blick nimmt und deren Bedeutung für die Wirtschaftsentwicklung betont. In dieser Arbeit wird die Finanzethik von Emunds aus der dem Postkeynesianismus entgegengesetzten Perspektive auf die Wirtschaft beleuchtet: aus der angebotsorientierten Sichtweise. Auch wird die Frage diskutiert, inwieweit sich der Postkeynesianismus als ein ökonomisches Komplement zur katholischen Soziallehre eignet. Im Rahmen dieses auf Finanzmarktfragen spezialisierten Beitrages ist jedoch eine ausführliche Gegenüberstellung von Postkeynesianismus und katholischer Soziallehre nicht möglich. Für die angebotsorientierte Ökonomik, z. B. in der Form der "Sozialen Marktwirtschaft", existiert schon eine lange und fruchtbare Auseinandersetzung mit der katholischen Soziallehre (z. B. Dölken 1992). In diesem Kontext muss besonders Joseph Höffner erwähnt werden, der sich als Vertreter der römischen Kirche intensiv mit der Sozialen Marktwirtschaft beschäftigt hatte (Franco 2015). Dieser Beitrag soll helfen, den Postkeynesianismus im Kontext der katholischen Soziallehre einzuordnen, wobei der Fokus vornehmlich auf Fragen der Finanzmärkte gerichtet ist. Es ist insgesamt für die katholische Soziallehre sehr lohnend, sich den bedeutenden und aktuellen Fragen zu stellen, welche die internationalen Finanzmärkte an eine Sozialethik herantragen.

Im nächsten Abschnitt wird dazu die postkeynesianische Ökonomik in Grundzügen vorgestellt. Darauf aufbauend wird die spezielle postkeynesianische Finanzmarktökonomik beschrieben, auf welche sich Emunds bezieht. Es werden im vierten Kapitel die
Schlussfolgerungen dargelegt, welche er für die konkrete Regulierung von Finanzmärkten zieht. Diese Finanzethik von Emunds wird danach einer kritischen Würdigung unterzogen: Zuerst wird sie in Kapitel 5 unter dem Aspekt betrachtet, wie sie das Problem der Ressourcenallokation behandelt. Im sich anschließenden Kapitel 6 wird die Frage behandelt, wie seine vorgeschlagene Finanzmarktregulierung die Unsicherheit in Bezug auf die Zukunft bewältigen will und wie sie mit dem Konflikt zwischen Systemstabilisierung und privater Haftung umgeht. Zum Schluss erfolgt ein Abgleich der postkeynesianischen Ökonomik mit Fragen, welche in der katholischen Soziallehre thematisiert werden: Dazu gehören Überlegungen zur Tugend der Sparsamkeit, zum Privateigentumsprinzip und zum Prinzip der Subsidiarität. Zum Schluss werden die Ergebnisse zusammengefasst und es werden Forschungspotentiale aufgezeigt.

\section{Die postkeynesianische Strömung in der Wirtschaftswissenschaft}

Der Postkeynesianismus ist nach der letzten großen und weltweiten Finanzkrise stärker wahrgenommen worden (Lavoie 2010). In Folge eines vermeintlichen Versagens der Mainstream-Ökonomik wurden alternative ökonomische Konzepte gesucht. Hier gibt es eine ganze Reihe alternativer Ansätze, welche ihre gegenläufige Ausrichtung zum ökonomischen Mainstream eint (Lavoie 2014: 7), und der Postkeynesianismus ist einer dieser Ansätze. Der ökonomische Mainstream wird vereinfacht ausgedrückt - durch die Verwendung neoklassischer Methoden identifiziert. Den Versuch einer Definition des "Mainstreams“ aus postkeynesianischer Sicht unternehmen Stockhammer und Ramskogler (2009: 334-338).

Die Frage, was unter „Postkeynesianismus“ genau zu verstehen ist, lässt sich allerdings nicht eindeutig beantworten. Lawson (1994: 503-504) vermutet, dass den Postkeynesianismus gerade seine Ablehnung des neoklassischen Ansatzes in der Ökonomie eint. Heise (2019: 5-8) sieht Gemeinsamkeiten der postkeynesianischen Ansätze in Fragen der philosophischen Fundierung, nämlich der ontologischen, und epistemologischen Opposition zum neoklassischen Mainstream: Das Say'sche Theorem sowie das Grenznutzenprinzip werden bestritten, und dies schließt ein, dass die von marktorientierten Ökonomen postulierte Selbstregulierung von Märkten nicht funktioniert. Auf der methodologischen Ebene wird die positivistische Theoriebildung im Sinne Friedmans (1953) abgelehnt. Friedmans Position besagt, dass Theorien eine starke Prognosekraft 
besitzen müssen, unabhängig von der Realitätsnähe der Annahmen. Dagegen wird bei den Theorien des postkeynesianischen Paradigmas auf Realitätsnähe Wert gelegt (Lavoie 2014: 12-15).

Es ist erklärtes Ziel der Postkeynesianer, der methodisch dominierenden Neoklassik mit den allgemeinen dynamisch-stochastischen Gleichgewichtsmodellen eine Alternative entgegenzusetzen. Dabei sind die Postkeynesianer darauf aus - im Gegensatz zu anderen keynesianischen Schulen wie bspw. der Neukeynesianer (Walterskirchen 2016: 413-415) - einen grundsätzlichen Gegenentwurf zur herrschenden Lehrmeinung zu formulieren. Postkeynesianer sehen sich insofern als authentische Interpreten von Keynes, als sie versuchen, ein konsistentes Theoriegebäude auf den von Keynes in der General Theory gelegten Prinzipien zu formulieren. Sie werfen anderen keynesianischen Strömungen vor, sich von Keynes nur Bruchstücke herauszupicken und diese lediglich als Erweiterungen in die neoklassische Theorie einzubauen. So hat demnach z.B. Paul Samuelson im Sinne einer neoklassischen Synthese mit keynes'schen Ideen gearbeitet (Davidson 2009: 19). Der „Neukeynesianismus“, der eine Mikrofundierung von makroökonomischen Theorien akzeptiert, aufgrund von Transaktionskosten nicht-markträumende Situationen betrachtet und damit „pseudo-keynesianische Ergebnisse“ erzielt, ist heute zum neuen Mainstream geworden (Stockhammer \& Ramskogler 2009: 336). Gegen diesen Neukeynesianismus grenzen sich die Postkeynesianer jedoch ab (Fontana \& Gerrard 2006: 53-54).

Während diese negative Abgrenzung des Postkeynesianismus einfach bestimmbar ist, stößt eine positive Definition der grundlegenden Annahmen eines postkeynesianischen Theoriegebäudes auf Probleme. Auch vor dem Hintergrund dieser internen Unbestimmtheit, aber vor allem aufgrund der Zweckmäßigkeit, werden hier nur drei der grundlegenden Postulate des Postkeynesianismus besprochen. Diese sind

(1) das Konzept der effektiven Nachfrage,

(2) die Ablehnung rationaler Erwartungen der Wirtschaftssubjekte und

(3) die Annahme grundsätzlich instabiler (Finanz-) Märkte.

\section{Das Konzept der effektiven Nachfrage}

Ewald Walterskirchen (2016: 417) stellt fest: „Das Prinzip der effektiven Nachfrage ist die tragende Säule des Postkeynesianismus. [...] Dieses Prinzip besagt, dass die effektive Nachfrage die Produktion und die Beschäftigung auch langfristig bestimmt." Hier findet eine Abgrenzung statt gegenüber einer angebotsorientierten
Wirtschaftspolitik, welche in der universellen Knappheit an Gütern das ökonomische Problem sieht, während der Postkeynesianismus in einer mangelnden effektiven Nachfrage das limitierende Moment des Wirtschaftswachstums ausmacht. Man geht also im Postkeynesianismus davon aus, dass ausreichend Produktionskapazitäten vorhanden sind, die aber aus unterschiedlichen Gründen nicht ausgelastet werden (Zorn 2016: 12). Daher ist die Unterbeschäftigung der Normalzustand. Das Produktionspotential, das wegen der Unterbeschäftigung brach liegt, gilt es mittels einer Nachfragepolitik zu heben und damit zu einem gewissen Gleichgewichtszustand zu kommen (Harcourt 2006: 145, 152). Das Say'sche Theorem wird umgedreht: Die Nachfrage schaffe sich ihr korrespondierendes Angebot. Die Vorstellung universeller Knappheit der Güter wird durch die „Reproduktionsfähigkeit“ ersetzt: Ausgenommen vom Faktor Land seien alle Produktionsfaktoren nur vorübergehend knapp (Lavoie 2014: 23). Die Allokation der vorhandenen Ressourcen sei also nicht das ökonomische Problem, sondern die Steigerung von Produktion und Wachstumsraten. Diese Vorstellung impliziert einen allgegenwärtigen Überfluss an Gütern.

Mit dem Prinzip der effektiven Nachfrage verknüpft ist die Frage nach der Kausalität zwischen Investieren und Sparen: Postkeynesianer gehen davon aus, dass die Investitionsnachfrage die Höhe der Ersparnisse bestimmt (Lavoie 2014: 35). Sie befürworten zudem eine arbeiterfreundliche Lohnpolitik, denn höhere Löhne führen wiederum zu einem Anstieg der effektiven Nachfrage. Somit haben hohe und gleiche Löhne eine wachstumsstabilisierende Funktion und sollen die unternehmerischen Profite letztlich sogar steigern (Stockhammer \& Ramskogler 2009: 339). Diese lohngetriebene Wachstumsstrategie steht im Gegensatz zu einer Vorstellung von Wirtschaftswachstum, welche eine steigende Arbeitsproduktivität, mithin also Investitionen in Humankapital sowie Forschung und Entwicklung, für die Voraussetzung des Wachstums der Ökonomie hält. Diese letztere dem ökonomischen Mainstream zugeordnete Ansicht wird kritisiert, weil sie vernachlässigt, dass gegenwärtige Wachstumsraten auch die potentiellen zukünftigen Wachstumsraten beeinflussen. Dies bedeutet, dass Lohnerhöhungen ursächlich zu einer erhöhten Arbeitsproduktivität führen (Lavoie \& Stockhammer 2012: 21).

\section{Die Ablehnung rationaler Erwartungen der \\ Wirtschaftssubjekte}

In den Schulen des ökonomischen Mainstreams herrscht gemäß den postkeynesianischen Autoren die Annahme vor, dass die Wirtschaftssubjekte rationale Erwartungen auf der Basis unbeschränkter Voraussicht 
auf die Zukunft bilden. Die Verhaltensökonomik versucht auf einer empirischen Basis zu zeigen, dass die realen Menschen keineswegs rationale Erwartungen bilden, sondern dass Menschen ihr Handeln z. B. auf Heuristiken aufbauen, also mithin irrational handeln, dennoch bewege sich die Standard-Verhaltensökonomik immer noch im Bereich des ökonomischen Mainstreams (Lavoie 2014: 15-16). Die Annahme rationaler Erwartungen setzt zugleich voraus, dass die Zukunft bekannt ist. Da dies aber eine sehr fragliche Annahme ist, zeigt sich hier ein realistischer Blick der Postkeynesianer auf die Wirklichkeit, denn bezüglich der Zukunft greifen sie die Unterscheidung von Risiko und Unsicherheit im Sinne Knights (1921) auf. „Risiko“ liegt genau dann vor, wenn für die Zukunft nur bekannte Szenarien vorliegen, denen eine quantitativ messbare Eintrittswahrscheinlichkeit zugeordnet werden kann, und diese Szenarien sind die „bekannten Unbekannten“. Nur in dieser Konstellation können rationale Erwartungen gebildet werden. Bei der „Unsicherheit“ sind nicht alle zukünftigen Szenarien bekannt; es gibt also die sog. „unbekannten Unbekannten“. Unsicherheit ist im Bereich des menschlichen Handelns der Normalfall und grenzt die Sphäre der Naturwissenschaften von der Sphäre menschlichen Handelns ab (Huerta de Soto 2013: 19). Die Vertreter des Postkeynesianismus bringen bei der Unsicherheit auch das Konzept der Nicht-Ergodizität von Davidson (1994) ins Spiel. Ein System ist dann ergodisch, wenn sich die Wahrscheinlichkeitsverteilung der Szenarien aus der vergangenen Entwicklung berechnen lassen. Nur in einem ergodischen System lassen sich demnach verlässliche Prognosen erstellen. Es gilt als ein Axiom des Postkeynesianismus, dass keine Ergodizität in der Realität vorliegt. Man kennt die wahre Wahrscheinlichkeitsverteilung von ökonomischen Sachverhalten nicht, weshalb aus den Daten der Vergangenheit und der Gegenwart keine Schlüsse für die Zukunft gezogen werden können. (Davidson 2009: 31).

Weil die ökonomische Realität im Postkeynesianismus als nicht-ergodisch betrachtet wird, spielt die Dimension der Zeit eine besondere Rolle. Die langfristige Entwicklung wird betrachtet als ein Aufeinanderfolgen vieler kleiner kurzfristiger Zeitabschnitte. Um die gesamte Entwicklung zu verstehen, muss man folglich den Übergang zwischen den einzelnen kurzen Zeitabschnitten erklären. Man erkennt hier, dass jedes Zeitintervall vom vorherigen Zustand abhängig ist. Es liegt also eine Pfadabhängigkeit der Entwicklung vor. Dies impliziert ebenfalls, dass die Zukunft nicht mit Hilfe von vergangenen Durchschnitten prognostizierbar ist (Lavoie 2014: 36). Daraus folgt eine besondere Zurückhaltung der Postkeynesianer bei der Verwendung ökonometrischer Methoden (Lavoie 2014: 37-8).

\section{Die Annahme grundsätzlich instabiler (Finanz-)Märkte}

Aus der postkeynesianischen Annahme, dass Preise nicht adäquat Knappheiten anzeigen können, folgt die These, dass freie Märkte mit freier Preisbildung Instabilität produzieren und dass daher staatliche Regulierung notwendig ist, um den Preisen eine gewisse Trägheit aufzuladen, welche die systemische Stabilität erhöht (Lavoie 2014: 25). Von Keynes übernimmt der Postkeynesianismus auch das Axiom der NichtSubstituierbarkeit aller ökonomischen Güter: Demnach gibt es nicht immer die Möglichkeit, auf ein passendes Substitut zurückzugreifen. Es kann also vorkommen, dass Märkte nicht "geräumt" sind, sich also nicht im Gleichgewichtszustand befinden (Davidson 2009: 30-31).

Die mikroökonomische Fundierung des neoklassischen Angebot-Nachfrage-Paradigmas baut auf der Annahme auf, dass die Angebots- und Nachfragekurven bestimmte Charakteristika aufweisen. Dazu gehört das nutzenmaximierende und rationale Verhalten der Wirtschaftssubjekte. Dann sind die abgeleiteten Angebots- und Nachfragekurven - abgesehen von speziellen Anomalien - so geneigt, dass sich ein Gleichgewicht einstellt. Postkeynesianische Autoren zeigen (Lavoie 2014: 50-51), dass selbst unter diesen Annahmen mehrere Gleichgewichtssituationen möglich sind. Wenn das der Fall ist, dann führt die unsichtbare Hand selbst, auch unter völlig freier Preisbildung, nicht zu einem stabilen Gleichgewicht und es können dann Phänomene wie eine unfreiwillige Arbeitslosigkeit entstehen (Fontana, Gerrard 2006: 51). Dies stellt besonders die neoklassische Annahme des methodologischen Individualismus in Frage: Basierend auf den individuellen Präferenzen, die sich meist am Modell des homo oeconomicus orientieren, werden in der Neoklassik Makrophänomene abgeleitet. Unter Wettbewerbsbedingungen stellt sich ein pareto-optimaler Zustand ein. Postkeynesianer wie auch andere „heterodoxe“ ökonomische Schulen hingegen versuchen, einen organisch-holistischen Ansatz zu verwenden (Lavoie 2014: 17): Hier wird ein koordiniertes Verhalten von Gruppen untersucht (Lavoie 2014: 52). Aggregate oder soziale Klassen wie „Arbeiter“, „Kapitalisten“ oder „Rentiers“ rücken in den Vordergrund der Analyse (Zorn 2016: 4). So wird versucht, die Interdependenzen zwischen den Individuen zu modellieren. Das klassische Beispiel hierfür ist das Herdenverhalten auf Finanzmärkten: Wenn sich eine irrationale Gier bei Finanzmarktteilnehmern einstellt, können die steigenden Wertpapierkurse bei weiteren Marktteilnehmern zur Ausbreitung der Gier führen. Auch in den postkeynesianischen Modellen geht man 
also zunächst von atomistischen Individuen aus, aber es werden Interaktionsregeln formuliert, welche eine komplexe soziale Struktur konstituieren. Danach können Makrophänomene abgeleitet werden, welche nicht allein vom individuellen Handeln her erklärt werden können.

Die Postkeynesianer gehen - mit diesen Überlegungen im Hintergrund - von verschiedenen makroökonomischen Paradoxien aus, welche von neoklassischen Ökonomen nicht gesehen werden können. Die Paradoxien entstehen in Folge nicht-intendierter Konsequenzen des individuellen Handelns und erklären mithin die Entstehung von unerwünschten Konjunktur- oder Finanzkrisen. Bekannter dürfte das Keynes'sche Sparparadox sein, welches erklärt, dass in Folge einer steigenden Sparneigung der Output sinkt (Zorn 2016: 14; Lavoie 2014: 18). Im Falle einer Konjunkturkrise würde das erhöhte Sparen also zu einer Verschärfung der Krise führen. Das Paradox der Kosten besagt, dass Reallohnsenkungen zu sinkenden Gewinnen der Unternehmen und damit zu einer erhöhten Arbeitslosigkeit führen. Das Paradox des Staatsdefizits widerspricht der Crowding-out-These der Neoklassik: Höhere Staatsverschuldung führt zu höheren Unternehmensgewinnen. Demnach wirken höhere Staatsdefizite ähnlich wie höhere Nettoexporte und höhere Staatsdefizite führen dann zu einem höheren gesamtwirtschaftlichen Output. Diese Paradoxien erklären, warum unregulierte Märkte immer wieder zu Krisen neigen, weshalb also die Marktwirtschaft eine inhärente Instabilität aufweist: Individuell rationales Verhalten kann zu kollektiv unerwünschten Ergebnissen führen. Der Preismechanismus kann die destabilisierenden Kräfte nicht ausreichend hemmen (Lavoie 2014: 34).

\section{Die postkeynesianische Sicht auf Finanzmärkte}

Die postkeynesianische Finanzmarktökonomik lässt sich an drei (oder in einer anderen Lesart vier) Paradoxien veranschaulichen (für die folgenden Ausführungen siehe Lavoie 2014: 19-22). Diese sind für die hiesigen Ausführungen wichtig, weil Emunds' Arbeiten auf diesen postkeynesianischen Überlegungen aufbauen.

Wenn ein Unternehmer seine Schulden reduzieren möchte, muss er möglicherweise kreditfinanzierte Investitionen unterlassen. Wenn dies mehrere Unternehmer gleichzeitig tun, dann kann die Abschwächung der Kapitalakkumulation die Profitabilität der Unternehmen senken. Weniger Kapitalakkumulation erschwert zudem die Innenfinanzierung. Folglich könnte es trotz gegenteiliger Absicht zu einem erhöhten Verschuldungsgrad der Unternehmen kommen. Ein ähnlicher Wirkungskanal würde auftreten, wenn Staaten sparen, um ihre Verschuldung im Verhältnis zum Output zu senken. Dieser Mechanismus würde auch dann auftreten, wenn Finanzinstitute ihre Verschuldungsquoten senken wollten: Der Verkauf von Assets („fire sales“) führt womöglich zu sinkenden Wertpapierkursen, wodurch die Aktivseite zu Lasten des Eigenkapitals abgewertet werden müsste. Der Verschuldungsgrad könnte entsprechend steigen. Alternativ könnte der Abbau der Verschuldung bei einer Bank dazu führen, dass Kreditnehmer ausfallen, weil innen eine Anschlussfinanzierung vorenthalten wird. Das belastet wiederum die Gewinnsituation der Bank. Gerade in einer Krisenphase wäre es für Banken individuell rational, ihre Risiken durch Kreditrationierung zu reduzieren, was aber den negativen makroökonomischen Effekt verstärkt.

Von Hyman Minsky, auf den sich Emunds (2018) explizit bezieht, stammt das Paradox der Ruhe. Eine Phase an den Finanzmärkten, die durch Wachstum und Stabilität gekennzeichnet ist, verwandelt sich demnach automatisch in eine spekulative BlasenÖkonomie. Dies liegt daran, dass - unter der Annahme der Unsicherheit über die Fundamentaldaten der Unternehmen - eine Reihe erfolgreicher Jahre zu niedrigeren wahrgenommenen Risiken von Finanzinstituten führt. Das heißt, dass die Statistik der vergangenen Kreditausfälle in einer langen Phase der Stabilität und Ruhe bspw. eine niedrigere Ausfallwahrscheinlichkeit von Krediten anzeigt. Mit der Zeit verschwindet die Erinnerung der Marktteilnehmer an Turbulenzen oder Rezessionen. Es werden höhere Verschuldungsquoten in den Finanzinstituten akzeptiert und die steigenden Gewinne bestätigen diese Strategie, sodass die Marktteilnehmer zunehmend Risiken akkumulieren. So kann sich eine Blasen-Ökonomie entwickeln, die den Keim ihres Zusammenbruchs in sich trägt.

Zuletzt wird von einem Paradox der Liquidität ausgegangen. Basierend auf der Effizienzmarkthypothese gehen neoklassische Ökonomen davon aus, dass die Preise an den Finanzmärkten die fundamentalen Daten gut widerspiegeln. Die Liquidität der Wertpapiere wird als gegeben angenommen. Postkeynesianische Ökonomen sind stärker auf die Liquidität fixiert und sehen auf zwei Ebenen ein Liquiditätsparadox. Gemäß der oben erwähnten fire-sales können die Märkte für liquide Wertpapiere in einer Krisensituation austrocknen. Wenn also Marktteilnehmer liquider werden wollen, so kann das dazu führen, dass liquide Assets zu nicht-liquiden Assets werden und die Liquidität der Akteure insgesamt sinkt. Ein zweites Liquiditätsparadox hängt mit Finanzinnovationen zusammen. Neue Wege der Unternehmensfinanzierung und neue Substitute für liquide 
Assets (bspw. Derivate) führen dazu, dass das Finanzsystem insgesamt illiquider wird. Dies führt zur Illusion, dass man jederzeit Zugang zu Zahlungsmitteln hat, und dass die Marktteilnehmer insgesamt weniger sichere Assets halten. Das Liquiditätsparadox hängt also mit einem Risikoparadox zusammen: Finanzinnovationen, welche Risiken besser handelbar machen, wie z. B. Verbriefungen oder verschiedene Derivate, führen zu erhöhten systemischen Risiken. Die scheinbare Quantifizierbarkeit von Risiken mithilfe von finanzmathematischen Methoden schafft eine Kontrollillusion, und dies führt zum Eingehen höherer Verschuldungsgrade. Dabei werden - auf der Ebene des Systems betrachtet - durch Derivate die Risiken insgesamt nicht weniger, sondern nur durch Kontrahentenrisiken ersetzt. Da jedoch die vielfältigen Beziehungen der Finanzinstitute nicht überblickt werden können, werden die Kontrahentenrisiken durch stärkeren Derivateeinsatz zunehmend unsicher. Lavoie (2014: 21) schreibt: „Indeed, even if the counterparty seems to be safe, the counterparty's counterparty may not be, and its failure may well spill over." Hier scheint wieder die wichtige postkeynesianische Annahme der Ablehnung rationaler Erwartungen durch: Die Zukunft ist unsicher, und diese Unsicherheit kann nicht quantitativ abgebildet werden. Die große Finanzkrise ist in diesen postkeynesianischen Denkstrukturen ein Ergebnis der Deregulierungsbestrebungen seit den 1980 er Jahren. Diese hatte man mit dem Argument durchgeführt, dass Märkte die vorhandenen Risiken am besten bepreisen können und dass die Marktdisziplin eine übermäßige Akkumulation von Risiken verhindern würde (Lavoie 2014: 25).

Die Postkeynesianer haben eine spezielle Sicht auf das Geldsystem. Besonders betont wird die Endogenität der Geldmenge (Stockhammer \& Ramskogler 2009: 339; Zorn 2016: 13 f.). Dies bedeutet, dass einerseits die Notenbank exogen einen Teil der Geldmenge produziert, dass aber andererseits der Großteil der Geldmenge durch die Kreditvergabe der Geschäftsbanken in den Umlauf kommt. Dadurch entsteht eine starke Prozyklizität der Geldmenge. Auch in der langen Frist ergibt sich die Geldmenge endogen. Dies impliziert, dass alleine die Nachfrage nach Geld die Geldmenge bestimmt, und die Geldnachfrage wiederum hängt vom Wirtschaftswachstum und dem daraus resultierenden Einkommen ab. Wie bei allen keynesianischen Richtungen wird von den Postkeynesianern besonders die Liquiditätspräferenz der Wirtschaftsakteure in einer von Unsicherheit geprägten Welt betont (Zorn 2016: 15). Der Zins ist ein Ergebnis dieser Liquiditätspräferenz. Es besteht stets die Gefahr, dass die Wirtschaftsakteure in einem pessimistischen und unsicheren Umfeld trotz hoher Zinsen ihr Kapital nicht investieren.

\section{Die politische Gestaltung internationaler Finanzmärkte bei Emunds}

\author{
Interpretation der Finanzmarktkrise 2008-2009
}

Die letzte große Finanzkrise wird bei Emunds ganz im Sinne der Instabilitätshypothese von Minsky erklärt (Emunds 2018); es wird also Marktversagen konstatiert. Dagegen verweisen ordnungspolitisch orientierte Ökonomen auf ein Staatsversagen im Bereich der Finanzmärkte (Michler \& Thieme 2009). Nach Emunds/Minsky liegt bei zu wenig bzw. schlecht regulierten Finanzmärkten das "Paradox der Ruhe“ vor. Die Ursache der Finanzkrise wird in einem schlechten Risikomanagement der Finanzmarktteilnehmer gesehen, die - auch vermittelt durch ABS-Transaktionen - zu hohe Risiken eingegangen sind. Minskys zentrale These ist, dass auf den Finanzmärkten zu viel Kapital vorhanden ist, welches die langanhaltenden Haussephasen bewirkt. Damit wird die Bedeutung der Kreditrationierung, also ein „zu wenig" an Kapital, als Krisenursache in den Hintergrund gedrängt. Mit Hilfe von extrapolativen Erwartungen nimmt Minsky an, dass sich eine expandierende Finanzökonomie von selbst weiter ausbreitet. Das Handeln der Wirtschaftsakteure führt also gerade nicht zu einem stabilen Gleichgewicht, sondern es bauen sich mit der Zeit Ungleichgewichte immer weiter auf. Die Haushalte verhalten sich genau wie die Finanzintermediäre prozyklisch: In guten Zeiten sinkt die Liquiditätspräferenz der Haushalte und damit steigt inr Verschuldungsgrad. Bei Finanzintermediären steigt in einem positiven Marktumfeld der Grad der Fristentransformation. In einer Krisensituation verhalten sie sich umgekehrt. Dieses Verhalten bewirkt die Übertreibung in der Boomphase und verschärft den Abschwung in der Rezession. In der Finanzkrise lagen diese Mechanismen sogar in verschärfter Form vor: Die Fristentransformation der Banken war durch Repo-Geschäfte sowie verschiedene Aktivitäten im Bereich der Schattenbanken besonders ausgeprägt. Dazu identifiziert Emunds eine sich seit den 1980er Jahren entwickelnde Abkopplung der Finanzmärkte von der Realwirtschaft. So wuchs der Anteil der Finanzwirtschaft an den am Sozialprodukt der verschiedenen Länder überproportional. Die starke Expansion der Finanzwirtschaft äußerte sich in einer Zunahme der Transaktionsvolumina und der Werte von Aktien und Derivaten, einer Vergrößerung des Eigenhandels, einer zunehmenden Fremdfinanzierung von Assetkäufen sowie einem Ausbau des Derivategeschäftes (Emunds 2014: 346-348). Gerade die Derivate führten zu einer Risikoblindheit, weil sie - ganz im Einklang mit dem 
von den Postkeynesianern beschriebenen Paradox der Liquidität - den Glauben an eine bessere Kontrolle der Risiken erhöhten, obwohl sie tatsächlich zu einer schlechteren Überschaubarkeit der Kontrahentenrisiken führten. So konnte es passieren, dass die Insolvenz einer mittelgroßen Investmentbank wie Lehman Brothers die Subprimekrise zu einer das Finanzsystem umspannenden Krise ausweitete. Diese Risikoblindheit wurde durch Anreizsysteme und innerorganisatorischen Renditedruck in den Finanzinstituten gefördert (Emunds 2014: 352-354).

\section{Die Rolle der Geschäftsbanken und des Kapitalmarkts}

Die Ursache für die Finanzmarktkrise liegt gemäß der postkeynesianischen Interpretation bei Emunds also in einem Überschuss an Kapital während der Boomphase. Dies wird durch verschiedene mikroökonomische Wirkungsketten erklärt. Da gibt es auf der einen Seite eine überdehnte Fristentransformation der Banken. Diese führe zu einer übermäßigen Anhäufung von Risiken in den Bankbilanzen. Besonders kritisch wird bei Emunds wie auch in der postkeynesianischen Literatur - die Geldschöpfung der Privatbanken gesehen. Die Kreditvergabe der Banken führt zur Geldschöpfung und deswegen geht die Erhöhung der Fristentransformation mit einer Erhöhung der Geldmenge einher. Die auf der Passivseite der Bankbilanz entstehenden Giroguthaben stellen wiederum einen Risikofaktor dar, weil die Gefahr des Bank Runs erhöht wird (Emunds 2014: 96 f.). Ein zweites Problem ist die überbordende Liquidität auf den Vermögensmärkten. Das durch die Ausweitung der Fristentransformation und der damit einhergehenden Geldschöpfung der Banken entstehende Geld fließt zu einem großen Teil auf die Vermögensgütermärkte. Dabei unterscheidet Emunds (2014: 98) einen direkten sowie einen indirekten Kanal: Direkt fließt das neugeschaffene Geld an die Vermögensgütermärkte, wenn Asset-Käufe mit Kredit finanziert werden. Indirekt fließt das Geld über die Erhöhung der Gewinne und Einkommen der Akteure der Realwirtschaft. Diese sind nun eher bereit, einen Teil der Einkommen zum Kauf von Aktiva zu nutzen. Insgesamt leistet also die Geldschöpfung durch Kreditvergabe die Finanzierungsfunktion für die steigenden Aktivapreise. Ein Rückkopplungseffekt entsteht dadurch, dass die gestiegenen Preise für Aktiva wegen ihrer Beleihungsfunktion eine erhöhte Kreditvergabe zulassen (Emunds 2014: 101).

Die sich seit den 1980er Jahren entwickelnde Angleichung von markt- und bankorientierten Finanzsystemen wird bei Emunds ausführlich betrachtet. Hier fand eine gegenseitige Befruchtung statt: Die Geschäftsbanken (commercial banks) des bankorientierten Finanzsystems nutzten zunehmend die Risikomanagementmethoden, welche in Investmentbanken entwickelt wurden. Mit diesen konnten die Kapitalanforderungen der Aufsicht umgangen werden, sodass eine Expansion der Risiken in den Geschäftsbanken möglich wurde. Dies geschah auch durch Kreditverbriefungen. Gleichzeitig haben umgekehrt die Geschäftsbanken das Geschäft der Investmentbanken befeuert, dadurch dass sie selbst im Eigenhandel Wertpapiere gekauft und den Käufern von Vermögensgütern Kredite zur Verfügung gestellt haben. Die Kreditvergabe der Geschäftsbanken war vorher mehr dadurch gekennzeichnet, dass das Geld an Unternehmen floss, die damit realwirtschaftliche Investitionen finanziert haben. Durch zahlreiche spekulative Transaktionen und Vermögensumschichtungen konnten die Investmentbanken viel Geld verdienen und haben zur Vergrößerung von Vermögenspreisblasen beigetragen (Emunds 2014: 350-352). Diese „Einkommen ohne Wertschöpfung" werden von Emunds deutlich kritisiert (Emunds 2013; 2015).

\section{Die Rolle der Zentralbank}

Die Zentralbank wird von den Postkeynesianern, so auch bei Emunds, ganz in Übereinstimmung mit dem „ökonomischen Mainstream“ betrachtet: Sie ist der Monopolist für das Geldangebot. In einer Aufschwungphase soll die Zentralbank durch eine antizyklische Zinspolitik die wirtschaftliche Aktivität dämpfen. Spiegelbildlich dazu sichert die Zentralbank die Liquidität der Finanzinstitute in Krisenzeiten; sie nimmt also die Rolle des Lender of Last Resort ein. Dies ist wegen der angestrebten antizyklischen Geldpolitik im postkeynesianischen Paradigma so auch notwendig: Postkeynesianer gehen davon aus, dass die Finanzmärkte inhärent instabil sind und deswegen der Stützung durch den Staat und die Zentralbank bedürfen (Emunds 2014: 84, 99, 105). Die mit der Rettungspolitik seit der Finanzkrise 2008-2009 einhergehende Moral Hazard-Problematik wird gesehen (Emunds 2014: 369). Die jüngeren Geldmengensteigerungen durch Zentralbanken („Quantitative Easing“ durch Käufe von Staats- und Unternehmensanleihen) werden im Werk von Emunds kaum kritisch beleuchtet, obgleich eine übermäßige und prozyklisch wirkende private Geldschöpfung durch den Bankensektor kritisiert wird. Emunds (2013: 19; 2015: 3) betont sogar, dass die gewinnorientierten Geschäftsbanken die treibende Kraft der Geld- und Kreditschöpfung seien.

\section{Die Rolle des Staates}

Eine Aufgabe des Staates sei es, die Finanzmärkte zu regulieren, damit die inhärenten Tendenzen zur Instabilität reduziert werden. Auch hier bewegt sich Emunds, genau wie die postkeynesianische Strömung, ganz im Rahmen der zeitgenössischen Standardökonomik. 
Die Regulierung soll den Finanzinstituten insbesondere in der Boomphase Beschränkungen auferlegen, sodass die Überhitzungstendenzen abgemildert werden können (Emunds 2014: 102). Dies ist vorrangige Aufgabe der Finanzaufsicht. Diese soll für eine Einlagensicherung sorgen, die gegebenenfalls auch durch eine staatliche Garantie gestärkt werden kann. Dazu kommt die Forderung einer prudenziellen Regulierung; dies läuft auf die Forderung einer Leverage-Ratio hinaus, was unten (vgl. Kap. 6) noch einmal vertieft wird. So sollen Risikomanagement und Eigenkapital der Finanzinstitute durch staatliche Vorgaben gelenkt werden. Emunds (2014: 358-360) kritisiert die tendenziell prozyklische Wirkung der Eigenkapitalregulierung von Banken. Die umstrittene Bevorzugung von Staatsanleihen durch eine nullprozentige Eigenkapitalunterlegung wird bei Emunds (2014: 109) nicht weiter kritisch reflektiert. Insgesamt wird eine "Globalsteuerung“ durch Staat und Zentralbank zur Stabilisierung der Finanzmärkte vorgeschlagen, d. h., dass die Finanzmarktregulierung nur auf der Makro-Ebene wirkt und private Anlageentscheidungen der Mikroebene möglichst nicht direkt beeinflusst werden sollten. Als Negativbeispiel erwähnt Emunds hier die Erfahrungen in verschiedenen kommunistischen Zentralverwaltungswirtschaften. Die Kreditvergabeentscheidung durch staatliche Stellen wird abgelehnt, weil sie meist mit einer zu lockeren Budgetrestriktion einhergeht und zu gesamtwirtschaftlicher Vergeudung von Ressourcen führt. Dabei werden das Fehlen privater Haftung und das Ausbleiben des Wettbewerbs kurz problematisiert (Emunds 2014: 110 f.).

\section{Reformvorschläge für das internationale Finanzsystem}

Auf Basis seiner Analysen leitet Emunds verschiedene Reformvorschläge für die globale Finanzwirtschaft $a b$. Es werden sechs verschiedene Maßnahmen vorgeschlagen, die auch an dieser Stelle eine kurze Würdigung erfahren sollen.

Zunächst soll das Eigenkapital der Banken durch höhere Quoten gestärkt werden und die Berechnung soll stark vereinfacht werden. Eine stärkere Haftung durch höhere Kernkapitalquoten hätte auch die notwendigen Anreizwirkungen, dass die Geschäftsmodelle auf eine langfristige Ertragsentwicklung ausgerichtet würden. In Aufschwungphasen sollen nochmals erhöhte Kapitalanforderungen die Kreditvergabe bremsen. Bei diesen Überlegungen geht Emunds auf einen Vorschlag ein, der nicht am Eigenkapital der Banken, sondern an deren Mindestreservepflicht ansetzt: Die Mindestreserveanforderungen werden erhöht, um eine restriktive Wirkung auf die Kreditvergabe zu erzielen. Eine detaillierte Ausarbeitung dieses Ansatzes findet aber nicht statt. Die Eigenkapitalregel soll einfach sein, d.h., dass die Risikogewichtung gänzlich wegfallen müsste. Interne Modelle der Finanzinstitute sowie andere Formen der Risikoquantifizierung, wie bspw. Ratings oder der Kreditrisiko-Standardansatz, würden dann nicht mehr benötigt (Emunds 2014: 372 f.).

Um eine kleinteiligere Struktur des Bankenmarktes herzustellen, wird eine Zerschlagung von großen Finanzkonzernen vorgeschlagen. Dies soll die Too-big-to-fail-Problematik lösen. Eine Alternative zur Zerschlagung wäre eine drastische Erhöhung der Eigenkapitalanforderungen für besonders große Institute. Dabei muss noch die Verflechtung der Institute untereinander berücksichtigt werden, denn auch schon der Konkurs eines mittelgroßen Institutes berge systemische Risiken (Emunds 2014: 374).

Die dritte Forderung enthält den Gedanken einer Trennung von Commercial- und Investmentbanking. Nach einer solchen Trennung ist es weiterhin möglich, die Teile mit Einlagengeschäft durch eine Einlagensicherung und Zentralbankgeld im Notfall abzusichern, damit der Zahlungsverkehr jederzeit gewährleistet ist. Die Investmentbanking-Aktivitäten sollen dagegen nicht abgesichert werden, damit hier eine volle Haftung für Verluste stattfindet. Auch die Risikoprämien, die solche Institute am Kapitalmarkt bezahlen müssen, sollen nach einer Trennung besser die wahren Risikoverhältnisse widerspiegeln. Die Investmentbanken können also gewisse riskante Geschäfte nicht mehr mit subventionierten Krediten tätigen. Eine solche Trennung des Investmentbankings vom Commercial-Banking reduziere besonders die systemischen Risiken im Finanzsystem (Emunds 2014: 375-377).

Durch eine selektive Erhöhung von Eigenkapitalanforderungen soll zudem gezielt bestimmten Preisblasen an den Vermögensgütermärkten entgegengewirkt werden. So könnten bspw. die Risikogewichte für Immobilienkredite erhöht werden, um einer Preisblase auf den Immobilienmärkten entgegenzuwirken. Die einfache Zinspolitik der Zentralbank reicht nicht aus, weil damit auch die Aktivitäten der Realwirtschaft eingeschränkt werden. Hier geht es also darum, finanzwirtschaftliche Rent-Seeking-Aktivitäten zu erschweren. In die gleiche Richtung geht die Einführung einer Finanztransaktionssteuer, welche ebenso vorgeschlagen wird. Diese soll kurzfristige Geschäfte stärker belasten als langfristige. Im Falle einer Preisblase an einem Teilmarkt wäre auch eine gezielte Erhöhung der Finanztransaktionssteuer in diesem Segment eine Alternative, um der Preissteigerung entgegenzuarbeiten (Emunds 2014: 378-381).

Um die Transparenz auf den Finanzmärkten zu erhöhen und um die Regulierungsumgehungen zu 
erschweren, soll ein globales Registrierungs- und Zertifizierungssystem für Finanzinnovationen eingeführt werden. Ergänzend dazu soll es eine Meldepflicht für alle OTC-Transaktionen von Derivaten geben. Diese Regeln gelten dann mitsamt allen übrigen Finanzregulierungen auch für Schattenbanken wie Hedgefonds oder Institutstöchter in Offshore-Zentren. Zuletzt wäre eine Stärkung der institutsübergreifenden Kommunikation, z. B. durch Berufsverbände, empfehlenswert, sodass diese mithelfen können, eine Unternehmenskultur zu fördern, welche das langfristige Denken in den Mittelpunkt stellt (Emunds 2014: 381-383).

\section{Diskussion der Finanzmarktökonomik bei Emunds: Die Frage der Ressourcenallokation}

Im weiteren Verlauf der Diskussion werden bestimmte Aspekte des Postkeynesianismus beleuchtet, welche auch für die Ethik wichtig sind. Weil sich Emunds auf diese ökonomische Schule bezieht, versuchen die folgenden Kapitel sowohl eine generelle kritische Würdigung des postkeynesianischen Ansatzes, als auch eine tiefere Durchdringung der Überlegungen zu Finanzmärkten bei Emunds. In Anlehnung an das Vorgehen bei Dölken (1992: 120 f.) werden nur Aspekte ausgewählt, die für die hier vorliegende Bewertung des Postkeynesianismus zweckmäßig sind. Dölken sieht bei der Beurteilung einer Wirtschaftsordnungskonzeption drei Punkte als besonders bedeutsam an: Diese sind die Produktion, die Allokation und die Distribution. Da die Perspektive hier nur auf den Finanzmarkt gerichtet ist, erfolgt zuerst eine Untersuchung hinsichtlich des Problems der Ressourcenallokation. Dem Postkeynesianismus wird im Folgenden eine „angebotsorientierte“ Perspektive gegenübergestellt, wovon sich der Postkeynesianismus explizit abgrenzt. Es geht dabei um die Axiome der ökonomischen Theorie: Die Knappheit ist wohl das zentrale Moment des Gegensatzes zwischen Postkeynesianismus und angebotsorientierter Ökonomik, was auch Lavoie (2014: 22) bestätigt, wenn er schreibt: „Scarcity is the fulcrum of neoclassical economics. [...] To proclaim the existence of an economy of plenty or an economics of abundance, as did Keynes, was to negate the foundations of orthodoxy." Hier soll also explizit diese "orthodoxe" Sicht in Bezug auf die Finanzmärkte zu Wort kommen und dem Postkeynesianismus gegenübergestellt werden, ohne dass sich die Diskussion an einer ganz bestimmten Version der angebotsorientierten Ökonomik (Neoklassik, Österreichische
Schule, Freiburger Schule, Institutionenökonomik etc.) orientiert.

\section{Die Ressourcenallokation in Euckens Ordnungstheorie und im Postkeynesianismus}

Was muss eine ökonomische Theorie leisten, um im Wettbewerb der Ideen eine ernstzunehmende Rolle zu spielen? Die Antwort auf diese Frage kann unterschiedlich ausfallen; in diesem Kapitel wird auf die diesbezüglichen Überlegungen von Walter Eucken rekurriert. Dieser beschreibt das Problem, mit dem die Wirtschaftswissenschaft konfrontiert ist, als die Frage der „zureichenden Lenkung des Wirtschaftsprozesses“ (Eucken 2004: 7 f.): „Ein Blick auf die Eigenwirtschaft zeigt uns [...] den entscheidenden Punkt; wenn der Leiter einer Eigenwirtschaft über einige Kubikmeter Holz Verfügungen zu treffen hat, so stellt er fest, welche Bedeutung die einzelnen Kubikmeter oder Stücke in den einzelnen Verwendungen gewinnen: Etwa beim Bau eines Stalles oder eines Wagens oder als Brennholz oder sonstwie. [...] Nur, weil der Leiter den Gesamtprozeß überblickt [...], kann er erfolgreich die einzelne Bewertung durchführen und die einzelne Anweisung geben. [...] Genau die gleiche Interdependenz und Einheit des Wirtschaftsprozesses besteht in der industrialisierten Wirtschaft. Niemand aber kann hier den Gesamtprozeß, wie er täglich abläuft, überblicken. [...] Wie kann dann aber überhaupt eine sinnvolle Lösung des Gesamtprozesses erfolgen?" Was bei Eucken mit der „Frage der zureichenden Lenkung des Wirtschaftsprozesses“ beschrieben wird, heißt in der modernen Ökonomik das „Allokationsproblem“. Eine ökonomische Theorie ist im Sinne Euckens dann zweckmäßig, wenn sie mit ihren Methoden eine Antwort auf das Allokationsproblem geben kann: Wann funktioniert der Wirtschaftsprozess so, dass eine größtmögliche Befriedigung der menschlichen Bedürfnisse gelingt? Der Postkeynesianismus antwortet auf diese Frage: Sobald die effektive Nachfrage ansteigt, erhöht sich auch der Output, welcher der Befriedigung der menschlichen Bedürfnisse dient. Im Beispiel von Eucken könnten die Mitglieder der Eigenwirtschaft ihre Nachfrage dadurch steigern, dass sie ihren Konsum erhöhen. Der Leiter der Eigenwirtschaft würde sehen, dass die Vorräte knapp werden und das Kommando geben, dass alle pro Tag länger arbeiten müssen. Dann ist die Forderung des Postkeynesianismus erfüllt: Die Nachfrage hatte sich erhöht, daraufhin hat der Leiter der Eigenwirtschaft, der den kollektiven Willen verkörpert, für eine steigende Nachfrage nach Arbeitsleistungen gesorgt, und dies erhöht den Output. So steigt insgesamt der Wohlstand der Gruppe. Die Voraussetzung dabei ist, dass der Leiter der Eigenwirtschaft um die Bedürfnisse der Gruppenmitglieder 
Bescheid weiß. Wichtig ist auch zu verstehen, dass die Gruppe einen Verzicht leisten musste, es entstanden mithin Opportunitätskosten: Zuerst musste mehr gearbeitet werden, damit die spätere Wohlstandssteigerung folgen konnte. Wie löst der Postkeynesianismus das Allokationsproblem in der Perspektive der Industriegesellschaft? Hier kann das Konzept der effektiven Nachfrage keine Wohlfahrtsmaximierung bewirken, weil über die Bedürfnisse der Wirtschaftssubjekte keine Informationen vorliegen. Hier gibt es den Leiter der Einzelwirtschaft nicht, also keine kollektive Intelligenz, welche alle Bedürfnisse bzw. Präferenzen kennen könnte. Eine Steigerung der effektiven Nachfrage im Sinne des Postkeynesianismus kann daher durchaus den Output in quantitativer Hinsicht erhöhen, aber ob der Güterzuwachs auch in qualitativer Perspektive mit den Bedürfnissen zusammenpasst, bleibt ungewiss.

Es ist eine grundsätzlich realistische Annahme der Postkeynesianer, davon auszugehen, dass es nicht ausgenutzte Produktionskapazitäten gibt. Sicherlich kann man diese aktivieren und erhält dadurch eine Steigerung der Güterproduktion. Dabei wird jedoch vernachlässigt, dass die Produktionsfaktoren wie Arbeit oder Kapital begrenzt sind. Wenn in einer bestimmten Branche die Produktion ausgeweitet wird, dann fehlen bspw. Arbeitskräfte, Energie, Immobilien oder Rohstoffe an einer anderen Stelle. Es entstehen immer Opportunitätskosten, welche das simple Kalkül der Ausnutzung von offenen Produktionskapazitäten möglicherweise gesamtwirtschaftlich unrentabel machen (Hazlitt 1946: 113 f.). Dieses Defizit des Postkeynesianismus hat zugleich eine wichtige ethische Dimension, weil eine mangelnde Allokationsleistung die Gefahr der Ressourcenvergeudung mit sich bringt (Nass 2020: 243 f.).

In den angebotsorientierten ökonomischen Schulen, welche eine universelle Knappheit der Güter annehmen, kann das Allokationsproblem mit der Interpretation von Preisen als Knappheitsindikatoren angegangen werden. So sieht es auch Eucken (2004: 8 f.), wenn er feststellt, dass alle Handlungen einer Person nur mit der Ausrichtung an einem Knappheitsmaß Sinn und Richtung erhalten. Dies gilt gleichfalls für eine große, industrialisierte und arbeitsteilig organisierte Gesellschaft. Das Wachstum führt dann zu einer Maximierung der Bedürfnisbefriedigung; es wird also ökonomischer Wert $(=$ Wohlfahrt) geschaffen. Sowohl beim Postkeynesianismus wie auch im angebotsorientierten ökonomischen Paradigma darf nicht vergessen werden, dass Wachstum alleine noch nicht die Vervollkommnung der menschlichen Natur garantiert; an dieser Stelle ist die Ethik als Ergänzung zur rein materiellen Betrachtung der Ökonomen gefragt (Utz 1993: 384).
Während also die angebotsorientierte Ökonomik von einer universellen Knappheit der Güter ausgeht und daher die Frage nach der effizienten Allokation derselben stellt, geht der Postkeynesianismus von einem allgegenwärtigen Überfluss aus und stellt dann die Frage nach der Hebung dieses Überflusses. An die Stelle der Knappheit wird die Fähigkeit zur Reproduktion gesetzt (Lavoie 2014: 22 f.). An dieser Herangehensweise des Postkeynesianismus ist richtig, dass es zumindest einen potentiellen Überfluss gibt. Die Tatsache, dass dieser nicht unmittelbar zu greifen ist, kann wiederum als Manifestation der Knappheit betrachtet werden. Es gibt darüber hinaus Güter, bei denen es nicht in der Macht des Menschen steht, ihr Angebot zu erweitern. In ganz spezieller Weise ist dies z.B. bei der Kryptowährung „Bitcoin“ der Fall: Hier gibt es unabänderlich nur 21 Mio. Einheiten (Erlei 2017). Bei derartigen Gütern müsste der Postkeynesianismus das Prinzip der Knappheit einräumen, so wie es auch bspw. beim Produktionsfaktor Land eingeräumt wird (Lavoie 2014: 23). Damit scheint die Annahme der universellen Knappheit der Güter allgemeiner zu sein als die Annahme eines potentiellen Überflusses. Willgerodt (2004: 27) sagt: „Der Mensch wirtschaftet aber, und er muß wirtschaften, weil er immer in einer Welt der Knappheit leben wird, und sei es auch nur der Knappheit seiner eigenen Lebenszeit."

Die postkeynesianische Sicht auf die ökonomische Realität vernachlässigt die Frage, was genau produziert werden soll. Der Postkeynesianismus kann keine adäquate Antwort geben auf das Problem der Erfassung von Konsumentenpräferenzen. In einer großen Industriegesellschaft sind individuelle Präferenzen überwiegend über Preise erkennbar. In einer Demokratie können daneben durch Wahlen kollektive Präferenzen ausgedrückt werden. Das Verfahren des Preismechanismus hat eine demokratische Note, weil jeder Marktteilnehmer durch seine Transaktionen andauernd an einer Abstimmung teilnimmt, was produziert werden soll, und dadurch seine Präferenzen offenbart. Genau diese von den Marktteilnehmer nachgefragten Güter werden wegen des Gewinnkalküls der Unternehmer tatsächlich produziert und erhöhen den Lebensstandard der Menschen: Die Markttransaktionen drücken ihre subjektive Wertschätzung aus (Rhonheimer 2020: 11, 14). Dieses Verfahren ist nicht demokratisch vollkommen, weil eine größere Kaufkraft die Produktion stärker beeinflussen kann. Wer mehr Geld hat, hat in diesem Sinne auch ein gewichtigeres Stimmrecht. Dennoch ist eine grundsätzliche Übereinstimmung der Produktion mit den Präferenzen der Marktteilnehmer gewährleistet. Der Postkeynesianismus sieht den Preismechanismus mit Skepsis. Die vom Postkeynesianismus für die 
Wirtschaftspolitik vorgeschlagene Nachfragesteuerung muss an verschiedenen Stellen in der Volkswirtschaft konkret wirksam werden. Also bestimmt die Wirtschaftspolitik explizit oder implizit, was nachgefragt wird, ohne dabei aber auf die wirklichen Präferenzen der Wirtschaftssubjekte zu reagieren. Problematisch dabei ist zudem, dass wirtschaftspolitische Interventionen immer auch von realen Politikern durchgeführt werden müssen. Diese verfolgen möglicherweise Eigeninteressen (Tietzel 1988: 87 f.). Staatliche Nachfrageaktionen laufen zudem stets Gefahr, von organisierten Interessen vereinnahmt zu werden (Schüller 2005: 67). Daher kann der Postkeynesianismus die Übereinstimmung der politisch erzeugten Nachfrage mit den Präferenzen der Wirtschaftssubjekte nicht sicherstellen, was im Marktsystem zumindest besser gewährleistet ist. Folglich ist die Güte der Ressourcenallokation einer auf postkeynesianischen Theorien aufbauenden Ordnungskonzeption unterlegen im Vergleich zur Ressourcenallokation auf der Basis einer angebotsseitig ausgerichteten Ordnungskonzeption.

\section{Die Ressourcenallokation auf dem Finanzmarkt bei Emunds}

Das postkeynesianische weitgehende Ausblenden der individuellen Präferenzen wird auch bei Emunds in seiner Sicht auf die Finanzmärkte deutlich. Er unterscheidet die Informationsverarbeitung und Ressourcenallokation einerseits durch Kapitalmärkte und andererseits durch Geschäftsbanken. Bezüglich der Kapitalmärkte gibt er die in der Finance-Literatur übliche Unterscheidung zwischen den Graden der Informationseffizienz wieder und kritisiert diese. Er stellt dem neoklassischen Ansatz, der davon ausgeht, dass ein gewisses Maß an Informationseffizienz auf den Finanzmärkten existiert, eine Minderheitenposition gegenüber, welche die Informationseffizienz auf Finanzmärkten stark anzweifelt. So wird die Existenz eines prinzipiell richtigen Modells zur Verarbeitung von Kapitalmarktinformationen und zur Erklärung des Fundamentalwertes eines Wertpapiers bestritten. Stattdessen gäbe es verschiedene kurzfristige wie langfristige Einflüsse, welche das Zustandekommen von Wertpapierkursen erklären. Dazu gehört z. B. eine psychologische Getriebenheit der Finanzmarktteilnehmer, welche die Aufwärts- wie auch die Abwärtsbewegungen verstärkt. Ebenfalls wird vorgebracht, dass die Marktteilnehmer ähnliche Bewertungsmodelle für Wertpapiere verwenden und dass durch computergestützte Handelstechniken sich gegenseitig verstärkende Trends entstehen können (Emunds 2014: 62). Insgesamt hält Emunds (2014: 64) die Informationsverarbeitung über den Kapitalmarkt für nicht ausreichend effizient für eine funktionierende volkswirtschaftliche Ressourcenallokation. Wohl ist die Bedeutung der Aktienmärkte nicht so groß für die Ressourcenallokation, während die Anleihemärkte wichtiger sind. Letztere hängen stark vom Einfluss der Ratingagenturen ab, was laut Emunds die Informationsverarbeitung kaum verbessert.

Der Informationsverarbeitung durch Geschäftsbanken wird aufgrund des Hausbankprinzips eine höhere Effizienz eingeräumt. Die Hausbank kann kostengünstiger und zielgerichteter Informationen über ihren Kreditnehmer einholen und auswerten. Diese Informationen weisen zudem eine höhere Qualität auf. Im Gegenzug zur besseren Informationslage gewährt die Bank dem Kreditnehmer eine gewisse Versicherung gegen Zahlungsschwierigkeiten. Dies verbessert insgesamt die Ressourcenallokation (Emunds 2014: 65).

Ob ein bankorientiertes Finanzsystem einem marktorientierten System überlegen ist, muss hier nicht weiter untersucht werden. Es ist im postkeynesianischen Paradigma konsequent, im marktorientierten System eine schwache Allokationsleistung zu erkennen. Das liegt an den inhärenten Instabilitäten und an den Paradoxien, die auf dem Finanzmarkt auftreten (vgl. Kap. 3). Diese Paradoxien betreffen aber das Bankensystem in gleichem Maße. Daher ist es vor dem Hintergrund des postkeynesianischen Ansatzes unplausibel, warum dem Bankensystem eine solche Überlegenheit attestiert wird: Das postkeynesianische Paradox des Deleveragings betrifft Finanzinstitute direkt. Das Paradox der Ruhe betrifft Finanzmärkte wie Finanzinstitute gleichermaßen. Das wird bspw. deutlich an der laxeren Kreditvergabe, wenn es lange keine Krise gegeben hat (Emunds 2018: 483), oder an der entsprechenden Ausdehnung der Fristentransformation bei einer gleichzeitig stattfindenden privaten Geldschöpfung (Emunds 2018: 479 f.).

Will man also die Ressourcenallokation auf Finanzmärkten verbessern, muss man die dort auftretenden angenommenen Irrationalitäten vermindern. Um Irrationalitäten auf dem Finanzmarkt oder bei Finanzintermediären zu identifizieren, wird ein nicht-irrationales Ideal vorausgesetzt. Andernfalls ist keine Steuerung gegen die Irrationalität möglich. In der Finanzökonomik beschäftigt sich die Behavioural-Finance-Theorie mit solchen Irrationalitäten. Letztlich basiert dieser Ansatz auf der Annahme einer neoklassischen Idealwelt, anhand der die Abweichung vom Ideal gemessen wird. Diese Abweichung entspricht der Irrationalität (Berg \& Gigerenzer 2010). Genau dieses neoklassische Ideal wird im Postkeynesianismus abgelehnt. Daher müsste ein anderer Weg zur Identifikation und Bewertung von Irrationalitäten vorgeschlagen werden. 
Bei Minsky wird nur anhand einer ausgebauten Theorie gesagt, dass es Irrationalitäten und Ineffizienzen gibt; so stellt er fest, dass die finanzielle Instabilität mit der Zeit zunimmt. Dies ist durchaus eine längerfristige Perspektive auf die Finanzmärkte (Emunds 2018: 483 f.). In diesem Paradigma ist es nicht möglich, den Grad der Instabilität zu bestimmen. Weil also quantitative Anhaltspunkte fehlen, kann auch keine kongruente Gegenmaßnahme bestimmt werden. Damit kann bei einer wirtschaftspolitischen Gegenmaßnahme nicht ex ante gezeigt werden, dass die Ineffizienz im System abgebaut wird, und es ist nicht ersichtlich, dass sich die Ressourcenallokation durch die vorgeschlagenen Maßnahmen verbessert.

Es zeigt sich hier wieder die Schwierigkeit des Postkeynesianismus, die Präferenzen der Wirtschaftsakteure angemessen in die Theorie einzubinden: Zunächst schafft ein Überschwang an den Finanzmärkten genau das, was von den Postkeynesianern gefordert wird, nämlich sehr viel effektive Nachfrage. Unternehmen bekommen leichteren Zugang zu Eigenkapital und zusätzliche Kredite. Privathaushalte und Staaten können sich ebenfalls günstiger finanzieren, und ihr erhöhter finanzieller Spielraum wird - wenn auch möglicherweise nicht vollständig - in der Realwirtschaft zu einer steigenden Nachfrage führen. Dies geschieht zunächst ohne Maßnahmen der Fiskal- oder Geldpolitik: Durch diesen Marktprozess kommen folglich die Präferenzen der Marktteilnehmer zum Ausdruck. Die schädlichen Auswirkungen dieses Aufblähens der Finanzmärkte, die „Rent-Seeking-Ökonomie“, werden klar gesehen (Emunds 2013). Wenn nun im postkeynesianischen Paradigma ein „irrationaler Überschwang“ identifiziert wird, dann heißt das nur, dass die zu befürwortende zusätzliche Nachfrage nicht dort ankommt, wo sie aus Sicht der postkeynesianischen Theorie ankommen sollte. Wie in der postkeynesianischen Ökonomik eine Bewertung der alternativen Kanäle für die Nachfrage vorgenommen wird, bleibt unklar.

\section{Diskussion der Finanzmarktökonomik bei Emunds: Stabilisierung unter Unsicherheit und private Haftung}

Ein weiterer Punkt, der näher zu untersuchen ist, ist die bei Emunds geforderte Stabilisierung von Märkten durch verschiedene Maßnahmen der Finanzmarktregulierung. Gleichzeitig wird davon ausgegangen, dass in der realen Welt Unsicherheit - statt Risiko - vorherrscht. An die Überlegungen zur Finanzmarktregulierung und-stabilisierung schließt sich die Frage an, ob das Prinzip der privaten Haftung ausreichend gewahrt ist.

\section{Finanzmarktregulierung unter Unsicherheit - ein innerer} Widerspruch?

Die große Bedeutung der Unsicherheit in der postkeynesianischen Ökonomik wurde in Kap. 2 angesprochen. Nicht nur hier, sondern auch bei der streng angebotsorientierten Österreichischen Schule der Nationalökonomie spielt die Unsicherheit - in expliziter Ablehnung des neoklassischen Risikobegriffs - eine wichtige Rolle (Mises 1949: 105-118). Diese Gemeinsamkeit stellt auch der Postkeynesianer Lavoie (2014: 29) fest. Die Österreichische Schule zieht aus dieser Annahme den Schluss, dass eine Regulierung nicht funktionieren kann (Dowd 1992: 685), während der Postkeynesianismus daraus einen Stabilisierungsauftrag ableitet.

Emunds (2014: 372) sieht, dass die Eigenkapitalregulierung vereinfacht werden muss. Ratings und interne Modelle sind geneigt, die wahre Ausfallwahrscheinlichkeit von Krediten nach einer langen Hausseperiode zu unterschätzen und damit das regulatorische Eigenkapital zu hoch auszuweisen. Daher ist von diesen Instrumenten keine stabilisierende Funktion zu erwarten. Folglich wird eine einfache Leverage-Ratio befürwortet, die aber sehr streng ist, d.h., dass sie Werte von 10-20\% oder mehr an hartem Kernkapital vorschreibt (Emunds 2014: 373). Eine Leverage-Ratio bietet Anreize zur Risikoerhöhung (Hartmann-Wendels 2016: 84 ff.), was auch Emunds einräumt. Dagegen verweist er darauf, dass die Banken zumindest gezwungen wären, einen relativ höheren Betrag an Eigenkapital vorzuhalten, wodurch die Eigenkapitalgeber stärker haften müssten. Deswegen hätten sie ein stärkeres Interesse an der Überwachung der Banken.

Wie ist eine Eigenkapitalvorschrift im Allgemeinen oder die Leverage-Ratio im Besonderen vor dem Hintergrund der Unsicherheit zu beurteilen? Die Unsicherheit führt dazu, dass Prognosen unmöglich sind. Es sind weder die denkbaren Szenarien für die Zukunft bekannt, noch können den Szenarien jeweils Wahrscheinlichkeiten zugeordnet werden. Bezogen auf das Bankgeschäft bedeutet das, dass das Insolvenzrisiko einer Bank nicht messbar ist. Es ist demnach nicht möglich, Kreditausfallraten aus der Vergangenheit herzunehmen und Extrapolationen durchzuführen, um zu einer Ausfallwahrscheinlichkeit für das Eigenkapital der Bank zu gelangen. Genau dies wird aber durch die Einführung einer Eigenkapitalvorschrift getan, denn auf dieser wissenschaftlichen Methode baut die gesamte risiko-basierte (nicht: unsicherheits-basierte) Regulierung auf (Heider 2015: 145): Die risikobasierten Instrumente durch bankinterne Modelle oder der 
Kreditrisiko-Standardansatz machen dies sehr feingliedrig; die Leverage-Ratio leistet dies sehr grob. Dennoch: Auch die Leverage-Ratio basiert auf einer Prognose. So ist es nämlich nicht irrelevant, ob man eine Eigenkapitalquote von 10, 20, 50 oder $80 \%$ vorschreibt. Weil die Zukunft ungewiss und das Verhalten der Marktteilnehmer nicht prognostizierbar ist, gibt es keine verlässliche Schätzung. Der Postkeynesianismus steht hier vor einem Dilemma: Einerseits wird die Notwendigkeit gesehen, die inhärent instabilen Finanzmärkte zu regulieren, andererseits bedürfte diese Regulierung gerade einer wissenschaftlichen Fundierung, welche im eigenen Paradigma abgelehnt wird. Es müsste zumindest eine Abwägung stattfinden zwischen den stabilisierenden Wirkungen der Regulierung und den negativen Begleiterscheinungen wie dem Ausmaß der Wettbewerbsverzerrung oder der Begrenzung des Intermediationspotenzials. Eine solche Betrachtungsweise kommt ohne ein gewisses Maß an Quantifizierung nicht aus. Um die Leverage-Ratio abschließend zu beurteilen, muss ihre Anreizwirkung untersucht werden. Emunds befürwortet sie wegen ihrer haftungserhöhenden Wirkung, während bspw. die Vertreter der Österreichischen Schule ihre allokationsverzerrende Wirkung betonen.

Ein weiterer Vorschlag zur Regulierung des Finanzsektors bei Emunds (2014: 378) fordert eine gezielte Erhöhung der Kapitalanforderungen für bestimmte überhitzte Branchen oder für systemrelevante Institute. Hierbei wird vorausgesetzt zu wissen, welche Branche sich gerade in einer Übertreibungsphase befindet. Hierzu heißt es bei ihm konkret: „Dass es auf einem Vermögensmarkt bzw. auf mehreren Märkten eine Preisblase gibt, kann z. B. aus einem außergewöhnlich starken Anstieg der Kredite, die für den Kauf dieser Vermögenswerte aufgenommen werden, geschlossen werden." Es ist zweifelhaft, ob mit wissenschaftlichen Methoden eine solche Prognose verlässlich erstellt werden kann. Es steht zu befürchten, dass die Bankenaufsicht einer „Anmaßung von Wissen“ im Sinne Hayeks (1974) unterliegt, wenn sie glaubt, die Zukunft an den Finanzmärkten besser prognostizieren zu können als die Marktteilnehmer. In der postkeynesianischen Ökonomik gibt es keine Anhaltspunkte, wie man eine Übertreibung identifizieren könnte. Hier stößt die Wissenschaft an die Grenze, welche die Unsicherheit vorgibt. Gerade der Postkeynesianismus betont die Nicht-Ergodizität in ökonomischen Systemen. Es kann also Pfadabhängigkeiten geben, sodass bspw. der Boom in einer bestimmten Branche am Finanzmarkt ihren Erfolg sogar noch bestärkt. Selbst wenn das Wissen darüber vorläge, ob sich ein Marktsegment in einer Übertreibungsphase befindet, so müsste noch nachgewiesen werden, dass die wirtschaftspolitische Gegenmaßnahme quantitativ angemessen und effizient ist.

\section{Die Trias von Stabilisierung, Haftung und dem „Paradox der Ruhe"}

Emunds sieht sehr deutlich die Problematik der fehlenden Haftung durch Moral-Hazard-Effekte. Er zitiert Hans-Werner Sinn, der die Privatisierung von Gewinnen bei gleichzeitiger Sozialisierung von Verlusten für den „mikroökonomischen Kern der Finanzkrise“ hält (Emunds 2014: 355). Im Kontext der von Emunds oft kritisierten Rent-Seeking-Ökonomie stehen die Moral-Hazard-Effekte im Fokus, denn diese führen gerade zu dem Phänomen des Rent-Seeking, bei dem Investitionen des Finanzsektors trotz fehlender volkswirtschaftlicher Wertschöpfung ein Einkommen generieren können. Die Ursachen dieses Phänomens werden nachvollziehbar identifiziert: Die staatliche Bailout-Politik garantiert die Gewinne im Zyklus der Finanzkonjunktur. Die durch die Geldschöpfung der Geschäftsbanken entstehende Geldmenge fließt direkt auf die Vermögensgütermärkte sowie in die Derivatemärkte. Davon profitieren auch die mit der Finanzwirtschaft verbundenen Dienstleister, wie z. B. Unternehmensberatungen oder M\&A-Vermittler. Die entstehenden temporären Seignorage-Gewinne kommen den Finanzinstituten zugute (Emunds 2013: 22-26).

Diese Überlegungen helfen dabei zu erklären, warum Emunds bestimmte Reformvorschläge für das Finanzsystem befürwortet. Mehrere Maßnahmen dienen dazu, die Haftung im System zu erhöhen und zugleich das Funktionieren des Wettbewerbsmechanismus sicherzustellen (vgl. Kap. 4):

- Trennbankensystem

- Erhöhung der Eigenkapitalanforderungen

- Zerschlagung von großen Finanzkonzernen

Ergänzend schlägt er verschiedene Instrumente vor, welche die Funktionsfähigkeit von Märkten wegen derer inhärenter Instabilität verbessern sollen:

- Zentralbank als Lender of Last Resort

- Einlagensicherung und Staatshilfen für Commercial Banks

- Reduktion der Teilreservehaltung

- Erschwerung der Regulierungsumgehungen

- Antizyklizität im Eigenkapitalpuffer

- gezieltes Entgegenwirken von Preisblasen

Im Bereich der Finanzmärkte gibt es einen Zielkonflikt zwischen der Durchsetzung der privaten Haftung und der Stabilität des Finanzsystems. Auf der einen 
Seite möchte man, dass Bankeigentümer für Verluste ihrer Bank haften. Dies heißt in letzter Konsequenz, dass eine geordnete Insolvenz möglich sein muss. Auf der anderen Seite legen die große Mehrheit der Finanzmarktökonomen, Zentralbanken und auch Regierungen großen Wert auf die Stabilität des Finanzsystems, welche mithin als „öffentliches Gut" bezeichnet wird (Michler \& Thieme 2009: 212). Der Prozess der Stabilisierung geht stets mit einem Verlust der privaten Haftung einher. Stabilisierung bedeutet, dass Banken im Krisenfall staatliche Liquiditäts- und Eigenkapitalspritzen bekommen. So kann eine „Rettung“ im Bereich der Finanzmärkte zum obersten Prinzip für Regierungen und Zentralbanken werden: Sobald die Stabilität des Systems in Gefahr gerät, wird mit zusätzlichen finanziellen Mitteln die geordnete Insolvenz für Finanzinstitute abgewendet, weil man davon ausgeht, dass die drohenden realwirtschaftlichen Verwerfungen zu schwerwiegend sind.

Alle von Emunds vorgeschlagenen Maßnahmen im Sinne des Haftungsprinzips führen zu einer Exante-Stabilisierung des Finanzsystems, während die Ex-post-Stabilisierung durch einen Lender of Last Resort oder Hilfen für Commercial Banks durch Rettungspakete das Haftungsprinzip aushebeln. Es liegt ein Zielkonflikt vor, der am Beispiel der Einlagensicherung deutlich wird: Der Zwang zur Einlagensicherung führt dazu, dass die Banken auf der Passivseite keine adäquaten Risikoprämien mehr bezahlen müssen, weil den Sparern die Anreize zum Monitoring weggenommen werden (Baltensperger 1990: 7). Folglich wird die disziplinierende Funktion des Marktes ausgeschaltet, die Bank kann höhere Risiken eingehen und die Sparer haften nun kollektiv für diese zusätzlichen Risiken (Kim \& Santomero 1988: 1219; Goebel 2013: 67). Die Einlagensicherung ist nicht dafür gedacht, im Falle einer Krise das gesamte Finanzsystem zu stützen; daher bleibt im Falle einer systemischen Krise die Notwendigkeit staatlicher Bailouts erhalten. Bei der Zentralbank als Lender of Last Resort verläuft die Argumentation ähnlich: Durch diesen Eingriff wird die Liquidität einer Bank jederzeit sichergestellt. Dieser Ex ante-Schutz führt bei der Bank zu Anreizen, in weniger liquide Assets mit höheren Erträgen zu investieren, da die Liquidität im Zweifelsfall von der Zentralbank bereitgestellt wird. Die höheren Renditen verbleiben bei der Bank, während die Liquiditätsrisiken auf die Gesellschaft abgewälzt werden. Ebenfalls haben Sparer wieder geringere Anreize, die Liquidität der Bank zu überwachen und es entstehen die bekannten Moral-Hazard-Effekte. Dies mache dann gemäß Emunds eine staatliche Regulierung erforderlich, welche einerseits die Wohlfahrtseffekte aus der Fristentransformation absichert, indem sie eine gewisse Stabilität sicherstellt, und andererseits die
Risikoübernahme der Finanzinstitute kontrolliert und im Zweifel begrenzt. Emunds bewegt sich soweit in Einklang mit der gegenwärtigen Finanzmarktökonomik. Eine echte Gegenposition wird von der Free-BankingSchule vertreten, welche den Zielkonflikt dadurch löst, dass keine Ex-post-Stabilisierung zugelassen wird, weil man davon ausgeht, dass ein unreguliertes Finanzsystem automatisch eigene stabilitätssichernde Institutionen ausbildet (Dowd 1988; Sechrest 1993).

Zuletzt wird die Frage aufgeworfen, ob die postkeynesianisch inspirierte Stabilisierungspolitik selbst dem „Paradox der Ruhe“ unterworfen sein kann. Heise (2019: 16) erklärt, dass die postkeynesianischen Vorschläge für die Wirtschaftspolitik genau darauf abzielen, Unsicherheit und Bewertungsvolatilitäten zu reduzieren, also für „Ruhe“ mit Hilfe interventionistischer Maßnahmen zu sorgen. Falls das „Paradox der Ruhe“ gilt, dann trägt jede Phase der Stabilität den Keim eines Ungleichgewichtes in sich. Je länger die Stabilität andauert, desto größer werden die Ungleichgewichte und desto wahrscheinlicher wird eine Entladung dieses Ungleichgewichtes in einer Krise. Müsste auf Basis des Paradoxes der Ruhe vielleicht gefolgert werden, dass der Staat für ein gewisses Maß an Unruhe und Volatilität im Finanzsystem sorgen sollte, damit jede Blasenbildung frühzeitig korrigiert wird? Gibt es statt des Paradoxes der Ruhe ein „Paradox der Stabilisierung“? Schüller (2009: 357) sieht ein solches Paradox der Stabilisierung. Er unterscheidet dabei einen ordnungspolitischen von einem interventionistischen Stabilisierungsansatz. Letzteren sieht er auch in dem Versuch, die gesamtwirtschaftliche Nachfrage zu steuern und hält diesen Ansatz für die Ursache der letzten großen Finanzmarktkrise (Schüller 2009: 359 ff.).

Dieses mögliche Paradox der Stabilisierung wird im Folgenden anhand des Beispiels einer möglichen „Zombifizierung" des Finanzsystems erklärt. Der Begriff der Zombifizierung entstand vermutlich zuerst im Kontext der langandauernden Stagnationsphase in Japan seit den 1990er Jahren. Hier hatten Banken, um Unternehmensinsolvenzen zu vermeiden, weiter Kredite an insolvente Unternehmen ausgereicht. Dies führte zu einem Verstoß gegen das Haftungsprinzip: Unrentable Unternehmen konnten so fortgeführt werden, was über Jahre hinweg stagnierende Wachstumsraten verursachte (Caballero et alii 2006). Dieselbe Logik lässt sich auch auf das Bankensystem übertragen: Sobald der Lender of Last Resort bzw. der Staat durch Rettungsaktionen das Finanzsystem stabilisiert, können Banken, die anderweitig hätten Insolvenz anmelden müssen, weiter ihre Geschäfte fortführen (Herok \& Schnabl 2018: 5 f.). Durch die geldpolitischen Maßnahmen wird die Situation verschlimmert, weil durch den niedrigen Zins 
die Ertragskraft des Bankensystems sinkt. Dies führt zu einer größeren Fragilität im Bankensystem, denn nun können Banken eine mögliche Zinserhöhung schwerer verkraften (Hoffmann \& Schnabl 2016: 460, 471; Krall 2020: 125-128). Damit bewirkt die Stabilisierung des Finanzmarktes in der mittleren und langen Frist das Gegenteil der beabsichtigten Wirkung und auch eine Stagnation in der Realwirtschaft, da die ZombieUnternehmen unbehindert weiterarbeiten können (Hoffmann \& Schnabl 2016: 455-457, 461).

\section{Der Postkeynesianismus im Verhältnis zur katholischen Sozialethik}

Der Postkeynesianismus ist eine sehr spezielle Strömung in der Wirtschaftswissenschaft, welche die Annahmen einer Mehrheit der Ökonomen in Frage stellt. Teile der christlichen Sozialethik sahen im 20. Jahrhundert eine weitgehende Übereinstimmung mit den zentralen Annahmen der Wirtschaftswissenschaft. Hier sei nur an die fruchtbare Auseinandersetzung von christlichen Sozialethikern beider Konfessionen mit den Vertretern des Ordoliberalismus erinnert (Franco 2015; 2017). Es stellt sich die Frage, ob die Voraussetzungen des Postkeynesianismus mit christlichen Vorstellungen kompatibel sind. Diese kann hier nicht abschließend beantwortet werden, weil der Fokus der Arbeit primär auf das Gebiet der Finanzmärkte gerichtet ist und die philosophischen und methodologischen Voraussetzungen des Postkeynesianismus nur streift. Eine erschöpfende Behandlung dieses Themas würde eine eigenständige Arbeit erfordern. Außerdem gibt es "die“ katholische Soziallehre, mit der der Postkeynesianismus verglichen werden müsste, so nicht. Daher können hier nur bestimmte Prinzipien der katholischen Soziallehre herangezogen werden, über die eine weitgehende Einigkeit herrscht, wobei selbst diese Prinzipien im Detail verschieden interpretiert werden können.

Der christliche Glaube und die christliche Morallehre treffen keine Aussagen zur Zulässigkeit ökonomischer Theoriesysteme. Emunds selbst ist katholischer Sozialethiker, daher sollte man von einer Kompatibilität ausgehen. Im Detail gibt es jedoch zumindest zu diskutierende Schwierigkeiten: Der Postkeynesianismus geht in seiner strengen Form von einem potentiellen Überfluss aller Güter aus und lehnt das Konzept der Knappheit ab. Umgekehrt ausgedrückt herrscht die effektive Nachfrage über Größen wie Sozialprodukt oder Beschäftigung, und in diesem Punkt sind sich Postkeynesianer einig (Fontana \& Gerrard 2006: 51 f.).
Eine Vereinbarkeit dieser Grundannahme mit christlichem Denken wird im Weiteren hinterfragt.

\section{Überfluss und Knappheit}

Der katholische Sozialethiker Joseph Höffner (1962: 142 f.) spricht eine klare Sprache bezüglich der Knappheit der Güter: „Die Erfahrung lehrt, dass die meisten Bedarfsgüter nicht in unbeschränkter Fülle, wie etwa die Luft, sondern nur begrenzt vorhanden sind; sie sind, verglichen mit den menschlichen Bedürfnissen, knapp. Auch unterliegen sie der Abnutzung und dem Verbrauch, so daß sich der Mensch immer wieder um ihre Beschaffung mühen muß. Dazu kommt, daß die Ansprüche des Menschen an die materielle Welt nicht wie beim Tier physiologisch gebunden sind, sondern - wegen seiner Geistigkeit - als unbegrenzt und unbegrenzbar bezeichnet werden müssen." Der evangelische Wirtschaftsethiker Wünsch (1927: 442 f.) beschreibt das Gesetz der Knappheit als einen permanenten Verzehr und die damit ständig drohende Gefahr der Knappheit. Sie setzt überhaupt erst das Wirtschaften in Gang, und im Gehorsam gegenüber Gottes Wille sollte der Mensch diesen Auftrag bejahen und seinen Sinn verstehen. Das Christentum hat sich eine gesunde Skepsis angeeignet, wenn es um den Zustand der materiellen Welt geht. Der christliche Gott-Vater hatte die Welt erschaffen, und er hatte sie "gut" geschaffen. Die ersten Menschen lebten demnach in einem Paradies, welches weder wirtschaftliche Not, noch Knappheit an Gütern noch den Tod kannte. Durch den Sündenfall fand eine Umkehrung des Verhältnisses des Menschen zur materiellen Welt statt: Von nun an musste der Mensch arbeiten, um sich selbst zu erhalten. Gleichzeitig war diese Arbeit mit großer Anstrengung verbunden (Höffner 1962: 119). Dies bezeugt die Stelle im Buch Genesis 3,17-18: „So ist verflucht der Ackerboden deinetwegen. Unter Mühsal wirst du von ihm essen alle Tage deines Lebens. [...] Im Schweiße deines Angesichts sollst du dein Brot essen, bis du zurückkehrst zum Ackerboden." Dies widerspricht scheinbar der postkeynesianischen Annahme eines prinzipiellen Überflusses an Gütern, der nur gehoben zu werden braucht. Auch diese Autoren gehen nicht davon aus, dass die Menschheit im Paradies lebt, denn dann wäre ökonomisches Fragen ohnehin überflüssig. Bei den Postkeynesianern ist dieser Überfluss potentieller Natur. Durch eine geeignete Nachfragepolitik muss der Überfluss aktiviert werden, um sich in steigendem Wirtschaftswachstum bzw. einer steigenden Gütermenge zu realisieren. Diese postkeynesianische Sicht geht also auch von einer Knappheit aus, man wählt hier lediglich eine andere Strategie zur Überwindung der Knappheit.

Der Unterschied zur angebotsorientierten Sicht liegt darin, dass die Knappheit laut den Postkeynesianern 
nicht in den Preisen sichtbar ist. Dagegen steht eine biblische Stelle, die sich als Preisänderung in Folge veränderter Knappheitsverhältnisse interpretieren lässt. Im Buch der Könige wird von einer großen Hungersnot durch eine Belagerung berichtet. Der Kopf eines Esels kostete dann achtzig Silberschekel und eine bestimmte Gewichtseinheit „Milchsterne“ kostete fünf Silberschekel (2 Kön 6,25). Die „Milchsterne“ waren dabei eine Nahrung für Notzeiten. Am Ende der Belagerung wurde das Lager der Feinde geplündert, und die Preise passten sich wiederum den neuen Bedingungen an: Jetzt kosteten ein Sea Feinmehl sowie zwei Sea Gerste nur noch einen Schekel (2 Kön 7,16). Der biblische Erzähler ist offensichtlich davon ausgegangen, dass hier die Preise als Knappheitsmaß für Nahrungsmittel zu interpretieren sind. Das Lehramt der katholischen Kirche sieht auch grundsätzlich, dass sich Preise als Knappheitsindikatoren interpretieren lassen. Der HI. Papst Johannes Paul II. schrieb in seiner Enzyklika Centesimus Annus, der freie Markt scheine das wirksamste Instrument für die Befriedigung der Bedürfnisse der Menschen zu sein (Johannes Paul II. 1991: Nr. 34). Dieser „freie Markt“ kann nicht anders interpretiert werden als ein System, welches die Preise der Güter als Knappheitsindikatoren zu nutzen weiß. So würdigt derselbe Papst den Gewinn „als Indikator für den guten Zustand und Betrieb des Unternehmens. Wenn ein Unternehmen mit Gewinn produziert, bedeutet das, daß die Produktionsfaktoren sachgemäß eingesetzt und die menschlichen Bedürfnisse gebührend erfüllt wurden." (Johannes Paul II. 1991: Nr. 35). Dabei lässt Johannes Paul II. es nicht unerwähnt, dass dieser Gewinn nicht verabsolutiert werden darf. Gleiches gilt für den freien Markt, denn es gibt Güter, die sich einer Bewertung durch Preise und der Logik des Tausches entziehen (Johannes Paul II. 1991: Nr. 40).

Das Lehramt greift auf eine lange theologische Tradition zurück, die auf den HI. Thomas von Aquin zurückreicht und die besonders in der spätmittelalterlichen Schule von Salamanca weiterentwickelt worden ist: Dies ist die Lehre vom gerechten Preis, der sich grundsätzlich am freien Markt unter Wettbewerbsbedingungen bildet (Roover 1958; Franco 2016: 42-44). Das aktuelle Lehramt von Papst Franziskus beruft sich nicht auf diese lange Tradition und sieht den Marktprozess erheblich kritischer als seine Vorgänger (z. B. Franziskus 2020: Nr. 168). Dabei wäre zu fragen, ob hier Franziskus unter dem Begriff „Markt“ als dem „Dogma des neoliberalen Credos“ das versteht, was mit der Lehre vom gerechten Preis gemeint ist.

\section{Sparen und Haushalten als christliche Tugenden}

Es ist sowohl auf einer biblischen wie auch auf einer moraltheologisch reflektierten Ebene beweisbar, dass privates Sparen eine christliche Tugend ist. Das Paradox des Sparens, welches der Postkeynesianismus behauptet, wird also im christlichen Denken so nicht anerkannt. Biblisch gesehen sei hier zunächst auf das Gleichnis vom anvertrauten Geld (Mt 25,14-30) verwiesen. Der Mann, der seinen Dienern das Geld anvertraut hatte, stellte innen seine Ersparnisse zur Verfügung. Er lobt die erfolgreiche Bewirtschaftung von den ersten beiden, während der dritte dafür getadelt wird, dass er das Talent vergraben hatte, anstatt es für Zinsen bei der Bank anzulegen. Im Alten Testament spricht das Buch der Chronik von den Vermögenswerten, die in der Schatzkammer des Tempels eingelagert wurden (1 Chr 26,20-28) oder von den königlichen Besitztümern (1 Chr 27,25-31; 2 Chr 11,5-12). Von staatlicher Verschuldung wird nicht berichtet. Eine weitere positive Darstellung des Sparens ist die Erzählung des alttestamentlichen Josef, der als Vorsteher von Ägypten in sieben Jahren des Überflusses einen großen Getreidevorrat anlegte. In den darauffolgenden sieben Jahren der Hungersnot, von welcher die ganze Welt betroffen war, verkaufte Josef das Brot an die Ägypter sowie an andere Länder (Gen 41,48-57). Mit dieser Strategie konnte Josef einen großen Gewinn erwirtschaften: Den Käufern ging das Geld aus, weil Josef die Einnahmen im Palast des Pharaos ansparte und nicht erneut in den Geldkreislauf brachte. Danach ließ sich Josef mit Vieh, später auch mit Ackerland und - dem modernen Denken völlig fremd mit der eigenen Versklavung bezahlen (Gen 47, 13-21). In einer ökonomischen Terminologie ausgedrückt hat Josef in einen Getreidevorrat investiert und dadurch für schlechte Zeiten vorgesorgt. Für dieses Verhalten wurde er durch einen üppigen Gewinn belohnt, denn in der Zeit der äußersten Knappheit konnte er einen sehr hohen Preis für das Getreide erzielen. Er hat mit diesem Sparvorgang das Volk der Ägypter wie auch die Völker anderer Länder vor dem Hungertod gerettet. Das Gegenteil des Sparens ist das Schuldenmachen, also das Leihen von Geld gegen Zins. Es wird gewarnt vor der Gefahr des Wucherzinses (Ps 15; Ez 18,8), aber gleichzeitig wird das Anhäufen von Reichtümern unter Zinsnahme zur Verteilung an die Armen gelobt (Dtn 15,8; Spr 28,8; Sir 29,1). Auch hier stellen die Autoren der Bibel die Vorsorge in ein positives Licht. Als letztes Beispiel soll die tüchtige Frau angeführt werden: Sie erzielt durch ihren Fleiß Gewinne, welche sie an die Armen weitergeben kann (Spr 31,13-20). Sie hat für den Winter vorgesorgt, indem sie einen Vorrat an wollenen Kleidern für ihre Hausangestellten angelegt hat (Spr 31,21-22).

Die Mäßigkeit ist eine christliche Tugend, welche den Einzelnen anhält, die sinnlichen Genüsse nur in einem angemessenen Maß anzustreben. Der Postkeynesianer 
Lavoie (2014: 22) stellt fest, dass religiöse Menschen tendenziell sparsamer sind. In der protestantischen Ethik ist die Bedeutung der Sparsamkeit in Kombination mit bestimmten Tugenden wie Fleiß hoch. Das bezeugt das Werk von Max Weber, der im protestantischen Raum ein höheres Wirtschaftswachstum feststellt, was er mit der protestantischen Ethik kausal verknüpft. Eine jüngere Studie stellt einen ähnlichen Einfluss im Umfeld zisterziensischer Klöster fest: Diese hatten eine stark ausgeprägte Leistungs- und Verzichtsethik, welche vermutlich das Bevölkerungswachstum in der umliegenden Gegend langfristig beflügelte (Andersen et al. 2016). In der abendländischen Tugendlehre wird die Mäßigkeit zu den vier Kardinaltugenden gezählt. Das Maß ist dann angemessen, wenn es weder ein „zu viel“ noch ein „zu wenig" gibt, d. h. die christliche Tugend der Mäßigkeit ist nicht rein negativ zu verstehen, als reiner Kampf gegen das Übermäßige oder Zuchtlose, und sie bedeutet auch mehr als eine Mäßigkeit im Bereich des Essens und Trinkens (Pieper 1960: 12-14). Besonders deutlich wird die christliche Wertschätzung der Mäßigkeit im Fastengebot: Hier soll durch ein zeitweises und stärkeres Einschränken der Genüsse eine Ordnung bzw. Zähmung der Leidenschaften bewirkt werden. Dies ist im christlichen Denken geboten, weil der Mensch durch die Ursünde stets zu einer Maßlosigkeit geneigt ist: Es findet in der menschlichen Seele ein andauernder Kampf zwischen „Fleisch“ und „Geist" statt (Gal 5,17). Man kann mit der Mäßigkeit alleine noch nicht eine christliche Wertschätzung des Sparens und sorgfältigen Haushaltens ableiten. Dazu bedarf es noch der Tugend der Klugheit (McCloskey 2006). Mäßigkeit ist eine „symmetrische“ Tugend, die wie alle anderen Tugenden „in der Mitte steht": Der Überfluss kann durch persönlichen Verzicht ohne Schwierigkeiten eingeschränkt werden. Ein Mangel aber kann nur durch Vorsorge behoben werden, indem also Vorräte für die Zeiten der Not angelegt werden. Dies ist dann ein Gebot der Klugheit. Dies gilt individualethisch wie auch sozialethisch: In einer Familie muss für die Zukunft vorgesorgt werden, dadurch dass man Ersparnisse bildet, und ebenso muss eine ganze Gesellschaft für Notzeiten Vorsorge treffen. Wilhelm Röpke (2009: 236 f.) unterscheidet die Eigenund die Fremdvorsorge. Eine Gesellschaft kann auf die Eigenvorsorge im Sinne des Aufbaus eines Staatsfonds verzichten und stattdessen in einer Notzeit Kredite am internationalen Kapitalmarkt aufnehmen. Das setzt voraus, dass eine entsprechende Bonität an den Märkten vorhanden ist. Überhaupt ist sogar eine Vorsorge auf kollektiver Ebene begrüßenswert; so wird es kaum möglich sein, bspw. die Norweger für ihren aus Öleinnahmen gespeisten Staatsfonds zu kritisieren. Ein Staatsfonds rohstoffexportierender Länder führt zu einer Absicherung gegen fallende Rohstoffpreise (Engerer 2016), also exakt gegen den Fall einer nationalen Notlage. So wie ein Individuum ein Vermögen anhäufen kann, um ein Einkommen aus Kapitalerträgen zu generieren, so kann das ein Staat ebenso. Im Sinne des Postkeynesianismus wird eine solche kollektive Vorsorge stattdessen die langfristige Entwicklung einer Nation verlangsamen. Dies ist tatsächlich dann gegeben, wenn durch den Sparprozess notwendige Investitionen unterlassen werden; in diesem Falle existiert ein gewisses Sparparadox. Ein staatliches Sparen kann also unter Umständen unklug sein, aber es kann nicht per se falsch sein.

Ist also der Postkeynesianismus mit der christlichen Tugend des Sparens vereinbar? Dies hängt von der Strenge ab, mit der die Existenz des Paradox des Sparens behauptet wird. Eine ökonomische Richtung, die das Sparen - sowohl individuell wie kollektiv - kategorisch ablehnt, kann niemals mit der christlichen Tugend der Sparsamkeit in Einklang gebracht werden. Eine mildere Form des Sparparadoxes, die vor den Gefahren einer übertriebenen und die wirtschaftliche Entfaltung bremsende Sparpolitik warnt, aber gleichzeitig den Wert des Sparens anerkennt, kann im christlichen Sinne akzeptiert werden. Dies schließt ein, dass einer Phase des Aufbaus von Staatsschulden auch grundsätzlich eine Phase des Abbaus dieser Verschuldung folgen muss. Die Schulden müssen dabei nicht zwingend vollständig getilgt werden, denn ein wachsendes Sozialprodukt verträgt auch eine höhere nationale Verschuldung.

Die Tugend befindet sich also auch hier „in der Mitte“: Christlich ist die Haltung, die eine Nachfragestimulierung nicht kategorisch ablehnt und insofern Züge des Postkeynesianismus akzeptiert. Eine unkritische Befürwortung aller Formen der Nachfragesteigerung, wie sie zumindest in einigen postkeynesianischen Schriften anklingt, steht nicht mit der christlichen Tugend der Mäßigkeit in Einklang.

\section{Die Frage nach dem Privateigentum}

Die Bedeutung des Privateigentums wird in der katholischen Soziallehre ununterbrochen diskutiert, was auch die Zentralität dieser Frage unterstreicht. Man reflektiert heute noch darüber, ob es möglicherweise einen urchristlichen Kommunismus gab (Reichel 2020). Bei den Kirchenvätern war die Frage nach einer Privateigentumsordnung zwar nicht explizit erörtert, aber die Diskussion war schon spannungsgeladen, wenn es um verwandte Themen wie Habgier oder Wucher ging (Nass 2013: 357). Bei Thomas von Aquin findet sich die erste systematisch erarbeitete Eigentumslehre (Rauscher 2008: 515). Seitdem das Lehramt, beginnend 
mit der Enzyklika „Rerum novarum“, konkret zu Fragen der Soziallehre Stellung nimmt, hat sich bis heute eine große Zahl an kirchlichen Dokumenten angesammelt, welche das Thema „Privateigentum" ausgiebig behandeln (Jayabalan 2014: 64-73; Emunds 2014: 37-39; Päpstlicher Rat für Gerechtigkeit und Frieden 2014: 140; Rauscher 2008: 518-521).

Die kirchliche Position zum Privateigentum wird hier nicht in extenso wiedergegeben und diskutiert. Es genügt der Hinweis, dass es grundsätzlich das Naturrecht auf Privateigentum gibt, wobei dieses - im Sinne der gleichzeitigen Sozialpflichtigkeit des Eigentums unter bestimmten Umständen aufgeweicht werden kann. Das Recht auf Privateigentum steht nämlich in einem Spannungsfeld zum fundamentalen Prinzip der universalen Bestimmung der Güter der Erde (Johannes Paul II. 1991: Nr. 6). Gerade darum muss sich eine ethische Reflexion über Finanzmärkte drehen: Sind die staatlichen Regulierungseingriffe, welche notwendigerweise in das Eigentumsrecht an den Produktionsmitteln eingreifen, angemessen?

Die Institution des Privateigentums spielt im Postkeynesianismus eine untergeordnete und unklare Rolle. Dennoch ist dies eine zentrale Frage für die Ausgestaltung einer Wirtschaftsordnung: So unterscheiden sich die beiden Pole der Wirtschaftsordnung, die Marktwirtschaft und die Zentralverwaltungswirtschaft, genau in diesem Punkt. Entsprechend bedeutsam ist diese Frage im Bereich der Finanzmärkte, und auch für die Ethik. Postkeynesianer stellen teilweise gravierende Eingriffe in das Privateigentum und die Vertragsfreiheit, aufbauend auf ihre ökonomische Theorie, als notwendig dar. Die damit einhergehenden Verluste an wirtschaftlicher Freiheit werden in dieses Kalkül mit einbezogen. Es stellt sich die Frage, ob es im Postkeynesianismus und bei Emunds im Besonderen eine innere Grenze für die Eingriffe in das Privateigentum gibt.

Das Lehrgebäude der Postkeynesianer baut ganz auf der Prämisse auf, dass die effektive Nachfrage zu steigern ist. Eine künstliche Steigerung der gesamtwirtschaftlichen Nachfrage kann nur mit einer Verletzung des Privateigentumsrechtes gedacht werden. Um dies zu zeigen, muss man sich die Wege vor Augen führen, welche die effektive Nachfrage praktisch erhöhen können. Im Postkeynesianismus geht man davon aus, dass der Großteil der effektiven Nachfrage durch die Investitionsentscheidungen von Unternehmen geschaffen wird (Zorn 2016: 12). Daneben gibt es die staatliche Nachfrage. Den Vorrang nimmt die Fiskalpolitik ein, aber auch die Geldpolitik ist von Bedeutung. Daneben beeinflusst der Staat die Lohnpolitik (King 2013: 495-498). Diese Politiken werden mitunter auch auf der Basis angebotsorientierter ökonomischer Theorien gefordert, aber sie haben im Postkeynesianismus eine nochmals gesteigerte Bedeutung.

Das Betreiben von Fiskalpolitik beeinflusst das Privateigentum. Fiskalpolitik bedeutet notwendigerweise, dass der Staat seine Ausgaben erhöht. Diese Ausgaben müssen finanziert werden, und das erfolgt in der Regel über Steuern. Hier wird deutlich, dass das Privateigentum der Steuerzahler betroffen ist. Auch bei der Finanzierung über Kredite ist der Steuerzahler betroffen, denn diese müssen in der langen Frist zurückgezahlt werden, was ceteris paribus einer erhöhten zukünftigen Steuerzahlung, möglicherweise erst für spätere Generationen, gleichkommt. Postkeynesianer würden hier einwenden, dass die Staatsausgabenerhöhung mit einem positiven Multiplikatoreffekt das Einkommen der gesamten Volkswirtschaft vergrößert. Dennoch liegt ein Umverteilungseffekt vor, denn es ist nicht klar, dass derjenige, der mehr Steuern bezahlen muss, auch ein entsprechend höheres Einkommen erhält. Von der angebotsorientierten Ökonomie wird betont, dass die expansive Fiskalpolitik mit einem Crowding-Out-Effekt einhergeht, welcher zusätzlich in die Verteilung des Privateigentums eingreift, weil die Unternehmer benachteiligt werden, die nur zu einem erhöhten Zinssatz ihre Investitionen durchführen können.

Die Geldpolitik kann durch ein Absenken des Zinses die effektive Nachfrage erhöhen. Dies geschieht über den Kanal der unternehmerischen Investitionen. Die künstliche Zinsänderung hat ebenfalls Umverteilungseffekte zur Folge. Hierzu gehören: ein überproportionaler Anstieg der Vermögensgüterpreise zu Lasten der Geldhalter, die Möglichkeit der Überraschungsinflation, welche die Sparer im Verhältnis zu den Schuldnern belastet, und die Erhöhung der realen Steuerlast aufgrund der kalten Progression (Polleit; Prollius 2010: 42-44; Hülsmann 2008: 46-49).

Bei der Lohnpolitik ist der Umverteilungseffekt einfach zu erkennen: Sobald der Staat die Löhne nach oben bewegt, wird in die Vertragsfreiheit der Unternehmer eingegriffen. Bei den Unternehmern, die diese erhöhten Löhne zu zahlen haben, entstehen höhere Kosten, und damit wird deren Recht auf Verwendung des Privateigentums angegriffen.

Nun kann es auch im Sinne der katholischen Soziallehre durchaus legitim sein, dass zur Förderung des Gemeinwohls Einschränkungen des Privateigentumsrechtes vorgenommen werden müssen. Dies bedarf aber einer Abwägung, denn als Prinzip gilt, dass das Privateigentum zu schützen ist. Erst wenn die Abwägung ergibt, dass die Vorteile aus der Verletzung des Privateigentumsrechtes dessen Nachteile übersteigen, kann ein staatlicher Eingriff legitimiert werden. In der Praxis ist diese Abwägung ein politischer Prozess, und 
auch in der Wissenschaft kann man über die Angemessenheit unterschiedlicher Meinung sein. Es kann daher nicht gesagt werden, dass die vom Postkeynesianismus vorgeschlagenen Interventionen per se mit der Soziallehre der Kirche unvereinbar seien. Problematisch ist dabei jedoch, dass die Verletzung des Privateigentumsprinzips in der postkeynesianischen Ökonomik kaum thematisiert wird: Dass die Verletzung des Privateigentums eine eigene Dynamik aufweisen kann, dass es sogenannte „Interventionsspiralen“ geben könnte (Kolev 2012: 50), wird im Postkeynesianismus nicht gesehen. Bedenklich ist zudem die ethische Komponente von staatlichen Verletzungen des Privateigentumsrechtes: Der Postkeynesianismus sieht nicht deren kollektivierenden und freiheitsberaubenden Charakter. Das Lehramt der Kirche wird dagegen nicht müde, die Personalität des Menschen zu betonen und vor dem Kollektivismus als Gefahr für die Freiheit und die Würde der Person zu warnen (Johannes Paul II 1991: Nr. 13). So ist der Mensch von seiner Natur her auf die Freiheit hin ausgerichtet (Johannes Paul II. 1991: Nr. 25), nicht auf den Status des von Bürokratien beherrschten Untertanen, und daher muss eine ökonomische Theorie auch diesen sozialen Aspekt hinreichend berücksichtigen, um mit der katholischen Soziallehre kompatibel zu sein.

\section{Das Prinzip der Subsidiarität}

Das Prinzip der Subsidiarität spielt im Postkeynesianismus keine Rolle - wobei dieser Befund auch für andere ökonomische Theoriesysteme gilt. Selbst bei Emunds, dem als katholischen Sozialethiker dieses Prinzip bekannt ist (Emunds 2010b: 4; 2014: 12), findet keine Auseinandersetzung mit der Frage statt, ob die politischen Gestaltungsvorschläge, die sich von der postkeynesianischen Fundierung her ableiten lassen, mit dem Subsidiaritätsprinzip kompatibel sind. Bei Utz (1964: 289) wird das Subsidiaritätsprinzip in seiner allgemeinsten Form folgendermaßen definiert: „Da das Gemeinwohl als Wohl von vielen personalen Wesen verstanden werden muß, ist die Freiheit des einzelnen ein Gut, welches zum Gemeinwohl gehört. Die Freiheit des einzelnen muß demnach nicht nur geduldet, sondern geschützt werden, sofern sie nicht dem Gemeinwohl zuwiderläuft." Utz (1964: 292) wendet dieses Prinzip auf die staatliche Tätigkeit an, und demnach ist „nur so Hilfe zu leisten, daß die Eigentätigkeit der „Zwischengesellschaften“ nicht erdrückt, sondern gestützt wird." In der Mitte des 20. Jahrhunderts hatte sich schon eine fortgeschrittene Auflösung gemeinsamer gesellschaftlicher Werte abgezeichnet. Bis heute hat sich dieser Trend ungebremst fortgesetzt. Dies verschärft die Notwendigkeit des Prinzips der Nichteinmischung des Staates (Utz 1964: 293 f.): Angesichts der Vielfalt der heutigen
Weltanschauungen besteht bei allen Tätigkeiten des Staates die Gefahr, dass bestimmte weltanschauliche Gruppen an ihrer privaten Entfaltung - möglicherweise auch ungewollt - gehindert werden. Auch Johannes Paul II. (1991: Nr. 48) betont die möglichen Funktionsstörungen in einer Gesellschaft, wenn das Prinzip der Subsidiarität nicht beachtet wird.

Wenn man diese Überlegungen auf die Ökonomik des Postkeynesianismus anwendet, kommt man zu dem Ergebnis, dass ein weitgehender Konflikt mit dem Subsidiaritätsprinzip vorliegt:

Im Bereich der Fiskalpolitik sind für den Postkeynesianismus weder das Individuum noch die „Zwischengesellschaften" bei der Steigerung der effektiven Nachfrage relevant. Das bedeutet, dass allein die zentrale Gewalt darüber entscheidet, in welchen Bereichen die Nachfrageerhöhung konkrete Gestalt annimmt. Es wäre denkbar, den Individuen oder anderen kleineren gesellschaftlichen Einheiten wie Kommunen, Vereinen oder Verbänden die Entscheidung über die Verwendung der Mittel zu überlassen.

Bei der Stimulation der effektiven Nachfrage durch Geldpolitik ist das Subsidiaritätsprinzip besser erfüllt. Der Lender of Last Resort muss logischerweise die zentrale Instanz sein (Gregg 2017: 180). Durch eine Senkung des Diskontsatzes können Banken günstigere Kredite vergeben. Dabei bleibt die Entscheidung der Kapitalverwendung bei den Banken und bei den Kreditnehmern. Bei Offenmarktgeschäften entscheidet dagegen ausschließlich die zentrale Instanz, also die Zentralbank, über die Verwendung der Mittel.

Es gibt im Postkeynesianismus kein Vertrauen in eine Selbstregulierungsfähigkeit des Bankensektors, weil die grundlegende Annahme der inhärenten Instabilität von Finanzmärkten die Sicht auf solche Potentiale verbaut. Es wäre im Sinne des Subsidiaritätsprinzips denkbar, dass die Banken- und Finanzbranche ihre Branchenregeln - wenn auch nur zum Teil - selbst entwickelt. Ein Beispiel einer selbst gestalteten Institution, welche ohne eine staatliche Vorgabe die Stabilität im System erhöht, ist das Einlagensicherungssystem im Verbund der Genossenschaftsbanken. Eine solche Form der Selbstregulierung wird im Postkeynesianismus und auch bei Emunds nicht betrachtet. Stattdessen wird akzeptiert, dass Regelungen auf höchster Ebene stattfinden. So ist es auch in der Praxis, wenn über supranationale Institutionen wie den Baseler Ausschuss für Bankenaufsicht Regeln entwickelt werden, welche über die jeweiligen Regulierungsbehörden in nationales Recht umgesetzt werden (Karassek 2013: 77 f.). Selbstregulierungspotentiale, welche bspw. von Uhde (2007) identifiziert werden, spielen im Postkeynesianismus keine Rolle. Bei Emunds (2014: 383) findet 
sich zumindest der Hinweis auf die Funktion, welche Berufsverbände für eine nachhaltige Unternehmenskultur einnehmen könnten. Es muss also ein - gleichwohl in Zukunft behebbarer - Konflikt mit dem Prinzip der Subsidiarität festgestellt werden.

\section{Zusammenfassung und Potentiale zukünftiger ethischer Reflexionen über Finanzmärkte}

Die Analyse hat aufgezeigt, dass in den spezifischen Fragen der politischen Gestaltung der Finanzmärkte einige Differenzen zwischen der Finanzethik bei Emunds und einer angebotsorientierten Ökonomik erkennbar sind. Diese Unterschiede wurden in den Kapiteln 5und 6 anhand wichtiger Kriterien wie der Ressourcenallokation herausgearbeitet. Die katholische Soziallehre dringt in diese Tiefe der Finanzmarktökonomik nicht vor. Daher kann aufbauend auf diesen „innerökonomischen“ Überlegungen eine weitere Reflexion anhand der Prinzipien der katholischen Soziallehre vorgenommen werden.

Für den Postkeynesianismus selbst wurde die Auseinandersetzung mit der katholischen Soziallehre im Kapitel 7 skizziert, wobei für eine umfassende Gegenüberstellung eine eigenständige Arbeit nötig wäre. Es können für die vier untersuchten Themenbereiche vorläufige Ergebnisse festgehalten werden.

(1) Überfluss und Knappheit als ökonomische Axiome: Eine Übereinstimmung der angebotsorientierten Sicht mit dem Axiom der Knappheit wurde aufgezeigt, und dass Preise als Knappheitsindikatoren fungieren, ist durch die Lehre vom gerechten Preis fester Bestandteil der katholischen Sozialethik. Der Postkeynesianismus kann - insofern er den angebotsorientierten Ansatz ergänzt - das Axiom eines Überflusses vertreten. Dadurch wird eine neue und bereichernde Perspektive auf die ökonomische Realität eingenommen, welche den Blick für nachfrageseitige Probleme schärft. Sobald der Postkeynesianismus dieses Axiom verabsolutiert, kann er nicht mit der katholischen Soziallehre kompatibel sein, weil sich die Knappheit als konstitutives Merkmal der Schöpfung nach dem Sündenfall aus der Bibel und der Lehrtradition der Kirche eindeutig ergibt.

(2) Sparen als individual- und kollektivethische Tugend: Wenn der Postkeynesianismus anerkennt, dass kollektives Sparen nicht in jedem Fall dem „Paradox des Sparens“ unterliegt, sondern dass das Auftreten dieses Paradox an
Bedingungen geknüpft ist, kann er die katholische Soziallehre bereichern. Eine angebotsorientierte Sicht könnte im Umkehrschluss ebenfalls Gefahr laufen, eine Austeritätspolitik zu verabsolutieren und damit die Mitte zu verlassen, welche eine jede Tugend nach Aristoteles fordert.

(3) Das Prinzip des Privateigentums: Der Postkeynesianismus spricht sich nicht für eine totale Kollektivierung der Eigentumsverhältnisse aus. Daher ist er hier mit der katholischen Soziallehre grundsätzlich kompatibel, weil diese im Gegenzug auch das Recht auf Privateigentum nicht verabsolutiert. Jedoch betont und stärkt die katholische Soziallehre das Prinzip des Privateigentums, während der Postkeynesianismus systematisch wirtschaftspolitische Maßnahmen vorschlägt, die das Privateigentum beschädigen. Emunds nimmt eine ausgewogenere Haltung ein, da er als katholischer Sozialethiker die Bedeutung des Privateigentumsprinzips kennt und Maßnahmen zur Stärkung des Haftungsprinzips hervorhebt.

(4) Das Subsidiaritätsprinzip: Der Postkeynesianismus beschäftigt sich zwar mit der Bedeutung von Institutionen, aber in keiner Weise systematisch mit dem Subsidiaritätsprinzip. Das Subsidiaritätsprinzip legt eine Kompatibilität mit dem methodologischen Individualismus nahe, den die angebotsorientierte Ökonomik vertritt. Der Postkeynesianismus grenzt sich durch einen stärker holistisch argumentierenden Ansatz davon ab. Auch bei Emunds spielt das Subsidiaritätsprinzip explizit keine Rolle. Eine Weiterentwicklung der postkeynesianischen Ökonomik in Richtung einer Integration des Subsidiaritätsprinzips könnte möglich sein. Dies setzt aber eine gewisse Relativierung des methodologischen Holismus voraus.

In der Gesamtschau muss eine unmittelbare Vereinbarkeit des postkeynesianischen Ansatzes in der Ökonomik mit den traditionellen Prinzipien der katholischen Soziallehre verneint werden. Die vorliegende Argumentation hat aufgezeigt, dass besonders das Prinzip des Privateigentums nicht ausreichend im Konzept des Postkeynesianismus berücksichtigt wird, während es als naturrechtliches Prinzip eine feste Säule der überlieferten katholischen Soziallehre bildet. Gewisse Relativierungen des Privateigentumsprinzips sind zwar notwendig, um das Gemeinwohl zu fördern; der Postkeynesianismus jedoch blendet die Problematik von Privateigentumsverletzungen systematisch aus. Damit 
kann die Frage, was dem Gemeinwohl wirklich dienlich ist, nicht adäquat beantwortet werden. Womöglich kann eine Weiterentwicklung des Postkeynesianismus dieses Problem zukünftig beheben und damit seine Anschlussfähigkeit an die katholische Soziallehre erhöhen. Ähnlich verhält es sich mit der Vernachlässigung der Signalfunktion der Preise: Das Gemeinwohl wird genau dann gefördert, wenn die ökonomischen Wünsche der Menschen bestmöglich erfüllt werden. Weil diese individuellen Präferenzen heute überwiegend über Tauschhandlungen und damit über Marktpreise kommuniziert werden, muss eine ökonomische Theorie diese als Daten ernstnehmen. Die postkeynesianische Annahme eines weitgehenden Marktversagens fordert entsprechend eine alternative Ermittlung der individuellen Präferenzen, welche jedoch bisher nicht in überzeugender Weise von den postkeynesianischen Autoren vorgelegt wurde. Somit bleibt es bei einer bloßen - und möglicherweise anmaßenden - Behauptung, die „richtige" Form der Nachfragesteuerung vorzuschlagen, welche die Wünsche der Konsumenten erfüllt und damit das Gemeinwohl nachhaltig fördert.

Die Ausführungen haben gezeigt, dass Emunds eine umfassende und originelle Reflexion über die Ethik der Finanzmärkte vorgelegt hat. Der Fortschritt in der Wirtschaftswissenschaft wird auch dadurch angetrieben, dass die herrschende Ansicht von Überlegungen auf der Basis unkonventioneller Prämissen herausgefordert wird. Dies hat Emunds mit seinem Bezug auf den Postkeynesianismus geleistet. Es bleibt der zukünftigen Diskussion überlassen, wie tragfähig dieser Ansatz sein wird.

\section{Literatur}

Andersen, Thomas B.; Bentzen, Jeanet; Dalgaard, CarlJohan; Sharp, Paul (2016): Pre-reformation Roots of the Protestant Ethic. In: The Economic Journal 127(604), S. 1756-1793.

Baltensperger, Ernst (1990): The Economic Theory of Banking Regulation. In: Furubotn, Eirik G.; Richter, Rudolf (Hg.): The economics and law of banking regulation. Occasional Papers Vol. 2. Saarbrücken, S. 1-21.

Berg, Nathan; Gigerenzer, Gerd (2010): As-If Behavioral Economics: Neoclassical Economics in Disguise? In: History of Economic Ideas 18(1), S. 133-165.

Caballero, Ricardo J.; Hoshi, Takeo; Kashyap, Anil K. (2006): Zombie Lending and Depressed Restructuring in Japan. NBER Working Paper Nr. 12129. Online verfügbar unter: https://www. nber.org/papers/w12129 [Abruf: 29.09.2020].
Für die vorliegende Arbeit, welche die Frage nach einer Regulierung der Finanzmärkte fokussiert, ist das Thema der Geldordnung untrennbar verknüpft. Auch hier gibt es bei Emunds Ansätze, denn er stellt die Frage, ob das auf der Teilreservehaltung basierende Bankensystem die Instabilitäten erhöht (Emunds 2010a: 112 f.). Grundsätzlich ließe sich das Thema der Geldordnung im Postkeynesianismus tiefer analysieren. Dies wurde in der vorgelegten Betrachtung nicht geleistet. Ebenso verhält es sich mit der internationalen Perspektive der Finanzwirtschaft, welche bei Emunds (2014: 217 ff.) eine bedeutende Rolle einnimmt: Fragen wie der internationale Kapitalverkehr, Devisenmärkte oder die internationale Regulierungsarbitrage blieben hier unberücksichtigt. Eine Auseinandersetzung mit diesen Themen wäre aber lohnenswert.

Emunds setzt richtungsweisende Akzente, welche die Debatte wohl auch in der Zukunft befruchten werden. Auch wenn viele Punkte in der Zunft der Ökonomen strittig bleiben, ist es wünschenswert, in der Ökumene Gemeinsamkeiten zu identifizieren, um als christliche Stimme nach außen wirken zu können. Die christliche Ethik hat die Verpflichtung, sich mit den eminent wichtigen finanzwirtschaftlichen Fragen weiter auseinanderzusetzen. Elmar Nass (2020: 56-80) hat jüngst einen Vorschlag erarbeitet, wie auch außerhalb der katholischen Sozialethik eine Integration mit anderen christlichen Konfessionen gelingen könnte. Es wäre eine Chance, diesen Ansatz auch im spezifischen Feld der Finanzmarktethik weiter zu entfalten und in die Praxis zu überführen.

Davidson, Paul (1994): Post Keynesian Macroeconomic Theory. Foundations for Successful Economic Policies for the Twenty-First Century. Cheltenham.

Davidson, Paul (2009): John Maynard Keynes. Basingstoke.

Dölken, Clemens (1992): Katholische Sozialtheorie und liberale Ökonomik. Das Verhältnis von Katholischer Soziallehre und Neoliberalismus im Lichte der modernen Institutionenökonomik. Tübingen.

Dowd, Kevin (1988): Automatic Stabilizing Mechanisms under Free Banking. In: Cato Journal 7(3), S. 643-659.

Dowd, Kevin (1992): The Case for Financial LaissezFaire. In: The Economic Journal 106(436), S. 679-687.

Engerer, Hella (2016): Staatsfonds in Zeiten niedriger Rohstoffpreise. DIW Roundup: Politik im Fokus Nr. 97. 
Emunds, Bernhard (2010a): Renditedruck der Finanzmärkte - schwere Zeiten für die Unternehmensethik. In: zfwu - Zeitschrift für Wirtschafts- und Unternehmensethik 11(2), S. 97-121.

Emunds, Bernhard (2010b): Ungewollte Vaterschaft. Katholische Soziallehre und Soziale Marktwirtschaft. In: Ethik und Gesellschaft (1) 2010: „Wem gehört die ,soziale Marktwirtschaft'?“ Herkunft und Zukunft einer bundesrepublikanischen Erfolgsformel. Online verfügbar unter: http:// www.ethik-und-gesellschaft.de/mm/EuG-1-2010_ Emunds.pdf [Abruf: 25.09.2020].

Emunds, Bernhard (2013): Die Finanzwirtschaft als Rent-Seeking-Ökonomie. In: Vierteljahreshefte zur Wirtschaftsforschung 82(4), S. 15-30.

Emunds, Bernhard (2014): Politische Wirtschaftsethik globaler Finanzmärkte. Wiesbaden.

Emunds, Bernhard (2015): „Finance" hat goldenen Boden - Über das Problem hoher Einkommen ohne Wertschöpfung. In: forum wirtschaftsethik 2015 (2), S. 2-7.

Emunds, Bernhard (2018): Did »it» happen again? Will »it« happen again? Eine Deutung der Globalen Finanzkrise und der heutigen Finanzwirtschaft im Lichte von Hyman P. Minskys Hypothese der finanziellen Instabilität. In: Leviathan 46(4), S. 474-492.

Erlei, Mathias (2017): Bitcoins. Blogbeitrag auf: Wirtschaftliche Freiheit. Das ordnungspolitische Journal. Online verfügbar unter: http:// wirtschaftlichefreiheit.de/wordpress/?p=21683 [Abruf: 01.12.2020].

Eucken, Walter(2004): Grundsätze derWirtschaftspolitik. 7. Aufl., Tübingen.

Fontana, Giuseppe; Gerrard, Bill (2006): The future of Post Keynesian economics. In: BNL Quarterly Review 59(236), S. 49-80.

Franco, Giuseppe (2015): Joseph Höffner und die Ordnungsethik. Eine Wechselbeziehung zwischen Katholischer Soziallehre und Wirtschaftsordnungen. In: ZfME - Zeitschrift für Marktwirtschaft und Ethik 3(2), S. 24-55.

Franco, Giuseppe (2016): Die Wirtschaftsanalyse und die Wirtschaftsethik der Schule von Salamanca. In: ORDO - Jahrbuch für die Ordnung für Wirtschaft und Gesellschaft 67, S. 33-74.

Franco, Giuseppe (2017): Glaube und Wirtschaft. Zur normativen Bedeutung des Ordo-Begriffes bei Walter Eucken. In: ZfME - Zeitschrift für Marktwirtschaft und Ethik 5(1), S. 23-38.

Franziskus (2020): Enzyklika Fratelli Tutti des Heiligen Vaters Papst Franziskus über die
Geschwisterlichkeit und die soziale Freundschaft. Vatikanstadt.

Friedman, Milton (1953): The Methodology of Positive Economics. In: Friedman, Milton (Hg.): Essays in Positive Economics. Chicago.

Goebel, Daniel J. (2013): Regulierungsmanagement in Banken. Eine Analyse von Handlungsfeldern zur Integration qualitativer Aufsichtsmaßnahmen in Bankstrategien am Beispiel der aktuellen Restrukturierungsgesetzgebung. Dissertation an der Heinrich-Heine-Universität Düsseldorf. Köln-Lohmar.

Gregg, Samuel (2017): Für Gott und den Profit. Eine Ethik des Finanzwesens. Christlich - marktliberal. Freiburg.

Harcourt, Geoffrey H. (2006): The Structure of PostKeynesian Economics. The Core Contributions of the Pioneers. New York.

Hartmann-Wendels, Thomas (2016): Die Leverage Ratio. Ausgestaltung, aufsichtliche Ziele, Auswirkungen auf die Geschäftspolitik der Banken. Gutachten für diedeutsche Kreditwirtschaft. Köln. Onlineverfügbar unter: https://die-dk.de/media/files/Gutachten Leverage_Ratio.pdf [Abruf: 01.10.2020].

Hayek, Friedrich A. von (1974): Die Anmaßung von Wissen. In: Viktor Vanberg (Hg.) (2004): Wirtschaftstheorie und Wissen. Aufsätze zur Erkenntnis- und Wissenschaftslehre. Friedrich A. von Hayek: Gesammelte Schriften in deutscher Sprache, A1. Tübingen, S. 87-98.

Hazlitt, Henry (1946): Economics in One Lesson. New York, London.

Heider, Felix (2015): Die risikobasierte Finanz markt regulierung im Kontext der Wissensteilung nach Hayek - eine wissenschaftstheoretische Analyse. Dissertation an der Heinrich-Heine-Universität Düsseldorf. Düsseldorf.

Heise, Arne (2019): Postkeynesianismus - Ein heterodoxer Ansatz auf der Suche nach einer Fundierung. Discussion Paper des Zentrums für Ökonomische und Soziologische Studien an der Universität Hamburg, September 2019. Online verfügbar unter: https://www.econstor.eu/ handle/10419/182463 [29.09.2020].

Herok, David; Schnabl, Gunther (2018): Europäische Geldpolitik und Zombifizierung. Austri-an Institute Paper Nr. 21.

Hoffmann, Andreas; Schnabl, Gunther (2016): Adverse Effects of Unconventional Monetary Policy. In: Cato Journal 36(3), S. 449-484.

Höffner, Joseph (1962): Christliche Gesellschaftslehre. Kevelaer.

Huerta de Soto, Jesús (2013): Sozialismus, Wirtschaftsrechnung und unternehmerische 
Funktion. Übersetzt von Marius Kleinheyer. Stuttgart.

Hülsmann, Jörg Guido (2008): The Ethics of Money Production. Auburn.

Jayabalan, Kishore (2014): The Catholic social encyclical tradition. In: Booth, Philip (Hg.): Catholic Social Teaching and the Market Economy. London, S. 56-77.

Johannes Paul II. (1991): Enzyklika CENTESIMUS ANNUS Seiner Heiligkeit Papst Johannes Paul II. an die verehrten Mitbrüder im Bischofsamt, den Klerus, die Ordensleute, die Gläubigen der katholischen Kirche und alle Menschen guten Willens zum hundertsten Jahrestag von RERUM NOVARUM. Verlautbarungen des Apostolischen Stuhles Nr. 101 vom 01.05.1991.

Karassek, Björn (2013): Kreditrationierung und Basel II. Begünstigen die Baseler Eigenkapitalvereinbarungen eine Rationierung der Kreditvergabe? Dissertation an der Universität Bremen. Berlin.

Kim, Daesik; Santomero, Anthony M. (1988): Risk in Banking and Capital Regulation. In: Journal of Finance 43(5), S. 1219-1233.

King, John E. (2013): A Brief Introduction to Post Keynesian Macroeconomics. In: Wirtschaft und Gesellschaft 39(4), S. 485-505.

Knight, Frank H. (1921): Risk, Uncertainty and Profit. Reprints of Economic Classics. New York. Online verfügbar unter https://www.mises. org/library/risk-uncertainty-and-profit [Abruf: 28.09.2020].

Kolev, Stefan (2012): Nicht wie viel Staat, sondern welcher Staat. In: Orientierungen zur Wirtschaftsund Gesellschaftspolitik 131(1), S. 48-52.

Krall, Markus (2020): Die bürgerliche Revolution. Wie wir unsere Freiheit und unseren Wohlstand erhalten. Stuttgart.

Lavoie, Marc (2010): Are we all Keynesians? In: Brazilian Journal of Political Economy 30(2), S. 189-200.

Lavoie, Marc (2014): Post-Keynesian Economics: New Foundations. Cheltenham.

Lavoie, Marc; Stockhammer, Engelbert (2012): Wageled growth: Concept, theories and policies. Conditions of Work and Employment Series No. 41. International Labour Office, Geneva.

Lawson, Tony (1994): The nature of Post Keynesianism and its links to other traditions: a realist perspective. In: Journal of Post Keynesian Economics 16(4), S. 503-538.

McCloskey, Deirdre N. (2006): Thrift as a Virtue, Historically Criticized. MPRA Paper No. 4088. Online verfügbar unter: https://mpra.ub.uni-
muenchen.de/4088/1/MPRA_paper_4088.pdf [Abruf: 01.10.2020].

Michler, Albrecht F.; Thieme, H. Jörg (2009): Finanz marktkrise: Marktversagen oder Staatsversagen? In: ORDO - Jahrbuch für die Ordnung für Wirtschaft und Gesellschaft 60, S. 185-221.

Mises, Ludwig von (1949): Human Action. A Treatise on Economics. The Scholar's Edition. 1998. Herausgegeben vom Ludwig von Mises Institute. Auburn.

Nass, Elmar (2013): Herkunft und Aktualität der christlichen Eigentumslehre. In: Die Neue Ordnung 67(5), S. 348-361.

Nass, Elmar (2020): Christliche Sozialethik. Orientierung, die Menschen (wieder) gewinnt. Stuttgart.

Päpstlicher Rat für Gerechtigkeit und Frieden (2014): Kompendium der Soziallehre der Kirche. 3. Aufl., Freiburg u.a.

Pieper, Josef (1960): Zucht und Maß. Über die vierte Kardinaltugend. 8. Aufl., München.

Polleit, Thorsten; Prollius, Michael von (2010): Geldreform. Vom schlechten Staatsgeld zum guten Marktgeld. Grevenbroich.

Rauscher, Anton (2008): Die christliche Lehre über das Eigentum. In: Rauscher, Anton (Hg.): Handbuch der Katholischen Soziallehre. Berlin, S. 511-522.

Reichel, Richard (2020): Urchristlicher Sozialismus, Solidarität und staatliche Umverteilung. In: Die Neue Ordnung 74(2), S. 112-125.

Rhonheimer, Martin (2020): Politik für den Menschen braucht weder "christlich“ noch „sozial“ zu sein. Austrian Institute Paper Nr. 29. Online verfügbar unter: https://austrian-institute.org/wp-content/ uploads/2020/03/Rhonheimer-Politik-für-denMenschen-AiPaper-Nr.-29-2020.pdf [Abruf: 05.10.2020].

Röpke, Wilhelm (2009): Jenseits von Angebot und Nachfrage. Nachdruck der 1958 erschienenen 1. Aufl., Düsseldorf.

Roover, Raymond de (1958): The Concept of the Just Price: Theory and Economic Policy. In: The Journal of Economic History 18(4), S. 418-434.

Schüller, Alfred (2005): Soziale Marktwirtschaft als ordnungspolitische Baustelle. Die Verbindung von „Freiburger Imperativ“ und „Keynesianischer Botschaft" - ein nationalökonomischer Irrweg. In: ORDO - Jahrbuch für die Ordnung von Wirtschaft und Gesellschaft 56, S. 61-75.

Schüller, Alfred (2009): Krisenprävention als ordnungspolitische Aufgabe. In: ORDO - Jahrbuch für die Ordnung von Wirtschaft und Gesellschaft 60, s. 355-388. 
Sechrest, Larry J. (1993): Free Banking. Theory, History, and a Laissez-Faire Model. 2008 neu herausgegeben vom Ludwig von Mises Institute, Auburn.

Stockhammer, Engelbert; Ramskogler, Paul (2009): Wie weiter? Zur Zukunft des Postkeynesianismus. In: Wirtschaft und Gesellschaft 35(3), S. 329-354.

Tietzel, Manfred (1988): Ethische und theoretische Probleme interventionistischer Wirtschaftspolitik. In: Cassel, Dieter; Ramb, Bernd-Thomas; Thieme, H. Jörg (Hg.) (1988): Ordnungspolitik. München, S. 77-105.

Uhde, André (2007): Selbstregulierungspotenzial in der Kreditwirtschaft. Eine vertragstheoretische und evolutionsökonomische Analyse. Dissertation an der Universität Bochum. Frankfurt am Main.

Utz, Arthur F. (1964): Sozialethik. Mit internationaler Bibliographie. I. Teil: Die Prinzipien der
Gesellschaftslehre. 2. Aufl., Heidelberg und Löwen.

Utz, Arthur F. (1993): Wohlstandsmehrung oder Sparen? In: Ockenfels, Wolfgang (Hg.) (1998): Ethik des Gemeinwohls. Gesammelte Aufsätze 1983-1997. Paderborn u.a., S. 382-387.

Walterskirchen, Ewald (2016): Neukeynesianismus und Postkeynesianismus: Was für ein Unterschied! In: Wirtschaft und Gesellschaft 42(3), S. 405-430.

Willgerodt, Hans (2004): Die Anmaßung von Unwissen. In: ORDO - Jahrbuch für die Ordnung von Wirtschaft und Gesellschaft 55, S. 25-35.

Wünsch, Georg (1927): Evangelische Wirtschaftsethik. Tübingen.

Zorn, Nicolas (2016): The Post-Keynesian Approach, A Relevant and Necessary Alternative. Working Paper. Online verfügbar unter https://www. researchgate.net/publication/303631716 [Abruf: 07.04.2020]. 